\title{
Solvmanifolds and noncommutative tori with real multiplication
}

\author{
Matilde MarColli
}

We prove that the Shimizu $L$-function of a real quadratic field is obtained from a (Lorentzian) spectral triple on a noncommutative torus with real multiplication, as an adiabatic limit of the Dirac operator on a 3-dimensional solvmanifold. The Dirac operator on this 3-dimensional geometry gives, via the Connes-Landi isospectral deformations, a spectral triple for the noncommutative tori obtained by deforming the fiber tori to noncommutative spaces. The 3-dimensional solvmanifold is the homotopy quotient in the sense of Baum-Connes of the noncommutative space obtained as the crossed product of the noncommutative torus by the action of the units of the real quadratic field. This noncommutative space is identified with the twisted group $C^{*}$-algebra of the fundamental group of the 3 -manifold. The twisting can be interpreted as the cocycle arising from a magnetic field, as in the theory of the quantum Hall effect. We prove a twisted index theorem that computes the range of the trace on the $K$-theory of this noncommutative space and gives an estimate on the gaps in the spectrum of the associated Harper operator.

1. Introduction

2. 3-Dimensional solvmanifolds and real quadratic fields

2.2. The topology of the 3 -manifold $X_{\epsilon}$

2.3. Action on $\mathbb{Z}^{2}$ 
4. Twisted group algebras and the magnetic Laplacian

4.1. Twisted group algebras 432

4.2. The noncommutative tori as twisted group algebras

4.3. Twisted group algebra of $S(\Lambda, V)$

4.4. The magnetic Laplacian

4.5. Discretized electron-ion interaction and Harper operators

4.6. Harper operators for noncommutative tori and for $S(\Lambda, V)$

4.7. Spectral theory and $K$-theory 438

5. Homotopy quotient and the Baum-Connes conjecture

5.1. $K$-theory of $C^{*}(S(\Lambda, V), \tilde{\sigma})$

5.2. $K$-theory and the twist

5.3. Thom isomorphism, homotopy quotients and Baum-Connes

6. Twisted index theorem, $K$-theory and the range of the trace

6.1. Spectral flow and odd Chern character

6.2. Twisted index theorem

6.3. Range of the trace

7. Isospectral deformations and spectral triples

7.1. The Connes-Landi isospectral deformations

7.2. Noncommutative solvmanifolds

7.3. Unitary equivalences 
8. Shimizu $L$-function and Lorentzian geometry

References

\section{Introduction}

In the 1970s, Hirzebruch formulated a conjecture, cf. [16], on the topological interpretation of certain special values of $L$-functions of totally real fields in terms of signature defects. The conjecture was proved in the early 80 s by Atiyah-Donnelly-Singer [2] and by Müller [24]. Hirzebruch's conjecture played an important role in the development of the Atiyah-Patodi-Singer index theorem [3], which in turn is a key ingredient in the proof [2] of the conjecture, extending the Hirzebruch-Riemann-Roch theorem to manifolds with boundary and relating the signature defect to the eta invariant. Geometrically, the link of an isolated singularity of the Hilbert modular variety associated to a totally real number field is given by a $(4 k-1)$-dimensional solvmanifold. The signature of the Hilbert modular variety is then computed by the APS theorem applied to the resulting manifold with boundary and the signature defects are computed by the eta invariant of the solvmanifold. The main step in the proof of [2] then consists of separating out the eta function of the signature operator on the solvmanifold into a part that recovers the Shimizu $L$-function of the totally real field and a residual part, which is shown not to contribute to the eta invariant.

We concentrate here on the simplest case, that of real quadratic fields, and we consider the question of whether the Shimizu $L$-function can be 
related in a similar way to a spectral geometry (in the sense of Connes' notion of spectral triples [11]) on a noncommutative torus with real multiplication by the same real quadratic field.

The motivation for such a question lies in the fact that the arithmetic contribution to the eta function in the argument of [2] appears to arise from a lower dimensional geometry which should correspond to the action of the units on a lattice in a real quadratic field, while the remaining part of the eta function can be attributed to the choice of a model, up to homotopy, of the same space. As we argue in more detail in the body of the paper, the kind of lower dimensional space that one would like to identify as supporting the arithmetic part of the eta function is a quotient, which is not a nice quotient in the ordinary sense, but which can be best described in terms of noncommutative tori with real multiplication.

The noncommutative tori associated to quadratic irrationalities have been extensively studied by Manin in [18] and subsequently by several authors. They have the special property of "real multiplication," derived from the presence of non-trivial self Morita equivalences. It is argued in [18] that the noncommutative tori with real multiplication should play a role for real quadratic field parallel to the theory of elliptic curves with complex multiplication in the case of imaginary quadratic fields. This makes it an interesting problem to study the geometric properties of this particular class of noncommutative spaces and their relation to the arithmetic of real quadratic fields.

We show in Section 5 that there is a close relation between the 3 -dimensional solvmanifold and the noncommutative torus with real multiplication. Namely, we prove that the first is the homotopy quotient, in the sense of Baum-Connes, of the noncommutative space given by the quotient of the latter by the action of the infinite group of units, Section 3. We also show that the 3-manifold can be identified with the pullback of the universal family of elliptic curves along a closed geodesic in the modular curve.

This interpretation as the homotopy quotient of a noncommutative space provides a geometric setting analogous to the one developed in the noncommutative geometry models of the quantum Hall effect [5], where the presence of a magnetic field makes the Brillouin zone of the lattice into a noncommutative torus. Here, the 3-dimensional solvmanifold is similarly related to a noncommutative space whose algebra of coordinates is the crossed product of the algebra of the noncommutative torus by the action of the units. This is obtained by twisting the group ring of the fundamental group of the solvmanifold by a cocycle, defined in terms of 
a magnetic potential. The noncommutative space is the resulting twisted group $C^{*}$-algebra, cf. Section 4 . As in the case of the quantum Hall effect, and in the noncommutative Bloch theory of electron-ion interactions, one obtains in Section 6 information on the spectral theory of the corresponding magnetic Laplacian by computing the range of the trace on the $K$-theory of the twisted group $C^{*}$-algebra. We prove a twisted index theorem which we use to compute the range of the trace using a spectral flow computation and the Baum-Connes conjecture, which is known to hold for the fundamental group of the 3-dimensional solvmanifold.

This way of passing from the 3 -dimensional solvmanifold to the associated noncommutative space is obtained in two steps. Viewing the 3-manifold as a fibration of 2-dimensional tori over the circle, one first replaces the fiber tori by noncommutative tori and then the mapping torus by the dual action of the units. We show in Section 7 that the first step can be seen as a case of the Connes-Landi isospectral deformations [13]. In particular, we prove that the Dirac operator on the 3-manifold induces in this way a Dirac operator on the noncommutative torus with real multiplication. A unitary equivalence as the one considered in [2] then factors this Dirac operator into a product of two operators, one of which has spectrum given by the norms $N(\lambda)$ of the lattice points $\lambda$ and recovers the Shimuzu $L$-function. In Section 7.4 we show how an adiabatic limit relates the Dirac operator on the 3-manifold to known differential operators on the noncommutative torus, obtained by considering the derivations along the leaves of the Kronecker foliations associated to the Galois conjugate elements $\theta$ and $\theta^{\prime}$ in the real quadratic field $\mathbb{K}=\mathbb{Q}(\theta)$.

Finally, we prove in Section 8 that the norms $N(\lambda)$ define the momenta of a Lorentzian Dirac operator on the noncommutative torus with real multiplication. The theory of spectral triples in Lorentzian signature is at present still under active development, and this provides a natural example where the arithmetic structure dictates how the Lorentzian geometry should be treated in the noncommutative context. We develop a framework for Lorentzian spectral triples over real quadratic fields, where the Galois involution of $\mathbb{K}$ provides a canonical choice of a Krein involution. In particular, we prove that, in passing from the indefinite Lorentzian geometry defined by the quadratic form given by the norm to the associated real Hilbert space, one can resolve the infinite multiplicities in the spectrum of the Dirac operator arising from the presence of a noncompact group of symmetries (the units of the real quadratic field). We show that the resulting operator on the real Hilbert space has the same eta function as the one coming from the adiabatic limit of the 3-dimensional geometry as in Section 7.4. This eta 
function is the product of the Shimizu $L$-function of the real quadratic field by a term that only depends on the fundamental unit.

\section{3-Dimensional solvmanifolds and real quadratic fields}

Let $\mathbb{K}=\mathbb{Q}(\sqrt{d})$ be a real quadratic field and let $\iota_{i}: \mathbb{K} \hookrightarrow \mathbb{R}$, for $i=1,2$, be its two real embeddings. We let $L \subset \mathbb{K}$ be a lattice, with $U_{L}^{+}$the group of totally positive units preserving $L$,

$$
U_{L}^{+}=\left\{u \in O_{\mathbb{K}}^{*} \mid u L \subset L, \iota_{i}(u) \in \mathbb{R}_{+}^{*}\right\}
$$

We denote by $\epsilon_{L}$ a generator, so that $U_{L}^{+}=\epsilon_{L}^{\mathbb{Z}}$. In the case where $L=O_{\mathbb{K}}$, the ring of integers of $\mathbb{K}$, then the generator $\epsilon=\epsilon_{L}$ is a fundamental unit. We consider the embedding of $L$ in $\mathbb{R}^{2}$ given by the mapping

$$
L \ni \ell \longmapsto\left(\iota_{1}(\ell), \iota_{2}(\ell)\right) \subset \mathbb{R}^{2} .
$$

We denote the range by $\Lambda=\left(\iota_{1}, \iota_{2}\right)(L)$. This is a lattice in $\mathbb{R}^{2}$. The action of $U_{L}^{+}$extends to an action on $\Lambda$ by

$$
\lambda=\left(\iota_{1}(\ell), \iota_{2}(\ell)\right) \longmapsto\left(\epsilon \iota_{1}(\ell), \epsilon^{\prime} \iota_{2}(\ell)\right)=\left(\epsilon \iota_{1}(\ell), \epsilon^{-1} \iota_{2}(\ell)\right) .
$$

\subsection{Semidirect products and solvmanifolds}

Let us denote by $V$ either the group $U_{L}^{+}$or a finite index subgroup thereof. As in [2], we consider the crossed product

$$
S(\Lambda, V)=\Lambda \rtimes_{\epsilon} V,
$$

where the action of $V=\epsilon^{\mathbb{Z}}$ on $\Lambda$ is induced by the action by multiplication on $L$. As shown in [2], these are discrete subgroups of the solvable Lie group

$$
S\left(\mathbb{R}^{2}, \mathbb{R}\right)=\mathbb{R}^{2} \rtimes \mathbb{R},
$$

with the action of $\mathbb{R}$ on $\mathbb{R}^{2}$ by the one parameter subgroup $\Theta_{t}(x, y)=$ $\left(e^{t} x, e^{-t} y\right)$ of $\mathrm{SL}_{2}(\mathbb{R})$. For $\epsilon>1$ and $\epsilon^{\prime}=\epsilon^{-1}<1$, the action of $V$ on $\Lambda$ 
is then generated by

$$
A_{\epsilon}=\left(\begin{array}{cc}
\epsilon & 0 \\
0 & \epsilon^{\prime}
\end{array}\right) \in \mathrm{SL}_{2}(\mathbb{R})
$$

We also consider the 3-dimensional solvmanifold obtained as the quotient

$$
X_{\epsilon}=S(\Lambda, V) \backslash S\left(\mathbb{R}^{2}, \mathbb{R}\right)
$$

with $\pi_{1}\left(X_{\epsilon}\right)=S(\Lambda, V)$.

\subsection{The topology of the 3-manifold $X_{\epsilon}$}

It is well known [2] that the 3 -manifold $X_{\epsilon}$ of (2.7) is a fibration over the circle $S^{1}$ with fibers that are 2 -tori and with monodromy given by the matrix $A_{\epsilon}$ of (2.6).

Lemma 2.1. The manifold $X_{\epsilon}$ has first homology

$$
H_{1}\left(X_{\epsilon}, \mathbb{Z}\right)=\Lambda /\left(1-A_{\epsilon}\right) \Lambda \oplus \mathbb{Z}
$$

Proof. The fundamental group is $\pi_{1}\left(X_{\epsilon}\right)=S(\Lambda, V)$. Consider the surjective map

(2.9) $\pi: S(\Lambda, V) \longrightarrow \Lambda /\left(1-A_{\epsilon}\right) \Lambda \oplus \mathbb{Z}, \quad \pi(\lambda, n)=\left(\lambda \bmod \left(1-A_{\epsilon}\right) \Lambda, n\right)$.

By writing

$$
A_{\epsilon}^{n}\left(\lambda^{\prime}\right)=\lambda^{\prime}-\left(1-A_{\epsilon}\right)\left(\lambda^{\prime}+A_{\epsilon}\left(\lambda^{\prime}\right)+\cdots+A_{\epsilon}^{n-1}\left(\lambda^{\prime}\right)\right)
$$

one sees that $\lambda+A_{\epsilon}^{n}\left(\lambda^{\prime}\right)=\lambda+\lambda^{\prime}$ modulo $\left(1-A_{\epsilon}\right) \Lambda$, so that $\pi\left(\lambda+A_{\epsilon}^{n} \lambda^{\prime}\right.$, $\left.n+n^{\prime}\right)=\pi(\lambda, n)+\pi\left(\lambda^{\prime}, n^{\prime}\right)$. Since commutators in $S(\Lambda, V)$ are of the form

$$
(\lambda, n)\left(\lambda^{\prime}, n^{\prime}\right)(\lambda, n)^{-1}\left(\lambda^{\prime}, n^{\prime}\right)^{-1}=\left(\left(1-A_{\epsilon}^{n^{\prime}}\right) \lambda-\left(1-A_{\epsilon}^{n}\right) \lambda^{\prime}, 0\right),
$$

we see that the homomorphism (2.9) has $\operatorname{Ker}(\pi)=\left[\pi_{1}\left(X_{\epsilon}\right), \pi_{1}\left(X_{\epsilon}\right)\right]$.

Corollary 2.2. The compact 3-manifold $X_{\epsilon}$ has cohomology

$$
H^{\text {even }}\left(X_{\epsilon}, \mathbb{Z}\right)=\mathbb{Z} \oplus \mathbb{Z} \oplus \operatorname{Coker}\left(1-A_{\epsilon}\right), \quad H^{\text {odd }}\left(X_{\epsilon}, \mathbb{Z}\right)=\mathbb{Z} \oplus \mathbb{Z} \text {. }
$$


Proof. By Poincaré duality, we have

$$
H^{2}\left(X_{\epsilon}, \mathbb{Z}\right) \cong H_{1}\left(X_{\epsilon}, \mathbb{Z}\right) \cong \mathbb{Z} \oplus \Lambda /\left(1-A_{\epsilon}\right) \Lambda
$$

and $H^{1}\left(X_{\epsilon}, \mathbb{Z}\right)=\operatorname{Hom}\left(H_{1}\left(X_{\epsilon}, \mathbb{Z}\right), \mathbb{Z}\right)=\mathbb{Z} \oplus \operatorname{Hom}\left(\Lambda /\left(1-A_{\epsilon}\right) \Lambda, \mathbb{Z}\right)$, so that

$$
H_{2}\left(X_{\epsilon}, \mathbb{Z}\right) \cong H^{1}\left(X_{\epsilon}, \mathbb{Z}\right) \cong \mathbb{Z} \oplus \operatorname{Hom}\left(\Lambda /\left(1-A_{\epsilon}\right) \Lambda, \mathbb{Z}\right)
$$

We have $\operatorname{Ker}\left(1-A_{\epsilon}\right)=0$, while $\operatorname{Coker}\left(1-A_{\epsilon}\right)$ is torsion, so that we obtain

$$
\begin{aligned}
& H^{0}\left(X_{\epsilon}, \mathbb{Z}\right)=\mathbb{Z}, \quad H^{1}\left(X_{\epsilon}, \mathbb{Z}\right)=\mathbb{Z} \\
& H^{3}\left(X_{\epsilon}, \mathbb{Z}\right)=\mathbb{Z}, \quad H^{2}\left(X_{\epsilon}, \mathbb{Z}\right)=\mathbb{Z} \oplus \operatorname{Coker}\left(1-A_{\epsilon}\right)
\end{aligned}
$$

\subsection{Action on $\mathbb{Z}^{2}$}

We recall the following description of the action of $A_{\epsilon}$ on $\Lambda$, which will be useful in the following, where we use twisted group $C^{*}$-algebras to describe noncommutative tori.

Lemma 2.3. In the basis $\{1, \theta\}$ of $\iota_{1}(L) \subset \mathbb{R}$, the action of the group $V=\epsilon^{\mathbb{Z}}$ is generated by the matrix

$$
\varphi_{\epsilon}=\left(\begin{array}{ll}
a & b \\
c & d
\end{array}\right) \in \mathrm{SL}_{2}(\mathbb{Z})
$$

with $\epsilon=a+b \theta$ and $\epsilon \theta=c+d \theta$. The conjugate elements $1 / \theta$ and $1 / \theta^{\prime}$ are the fixed points of $\varphi_{\epsilon} \in \mathrm{SL}_{2}(\mathbb{Z})$ acting on $\mathbb{P}^{1}(\mathbb{R})$ by fractional linear transformations.

Proof. As we have seen in (2.3), the action of $V$ on $\Lambda$ is given by

$$
A_{\epsilon}:\left(n+m \theta, n+m \theta^{\prime}\right) \longmapsto\left(\epsilon(n+m \theta), \epsilon^{\prime}\left(n+m \theta^{\prime}\right)\right)
$$

with $\epsilon \epsilon^{\prime}=1$. In particular, for $m=0$ and $n=1$ this gives $\epsilon \in \iota_{1}(L)$ and $\epsilon^{\prime} \epsilon$ $\iota_{2}(L)$. Thus, we can write $\epsilon=a+b \theta$, for two integers $a, b \in \mathbb{Z}$. Similarly, the element $\epsilon \theta$ can be written in the form $\epsilon \theta=c+d \theta$. Thus, the action of 
$A_{\epsilon}^{k}$ on $\Lambda$ can be described equivalently as

$$
(n, m) \longmapsto(n, m) \varphi_{\epsilon}^{k}, \quad \text { with } \varphi_{\epsilon}=\left(\begin{array}{ll}
a & b \\
c & d
\end{array}\right) \in \mathrm{SL}_{2}(\mathbb{Z})
$$

The second statement follows immediately since

$$
\theta^{-1}=\frac{\epsilon}{\epsilon \theta}=\frac{a \theta^{-1}+b}{c \theta^{-1}+d}
$$

We obtain in this way two corresponding identifications $S(\Lambda, V)=$ $\mathbb{Z}^{2} \rtimes_{\varphi_{\epsilon}} \mathbb{Z}$, by mapping $\left(\lambda, \epsilon^{k}\right)$ to either $\left(\lambda_{1}=n+m \theta, k\right)$ or $\left(\lambda_{2}=n+m \theta^{\prime},-k\right)$.

\subsection{Solvmanifold and Hecke's lift of geodesics}

For $\Gamma=\mathrm{SL}_{2}(\mathbb{Z})$ and $X_{\Gamma}=\Gamma \backslash \mathbb{H}$ the modular curve, let $\mathcal{U}_{\Gamma} \rightarrow X_{\Gamma}$ denote the universal family of elliptic curves over the modular curve, where the fiber over $\tau \in X_{\Gamma}$ of $\mathcal{U}_{\Gamma}$ is the isomorphism class of the elliptic curve $E_{\tau}=$ $\mathbb{C} /(\mathbb{Z}+\tau \mathbb{Z})$.

Suppose given a lattice $L$ in a real quadratic field $\mathbb{K}$ and let $\{1, \theta\}$ be a basis for $L$, with $\theta^{\prime}$ the Galois conjugate of $\theta$ under the Galois involution of $\mathbb{K}$ over $\mathbb{Q}$.

We denote by $\gamma_{\theta, \theta^{\prime}}$ the infinite geodesic in the hyperbolic plane $\mathbb{H}$ with endpoints $\theta, \theta^{\prime} \in \mathbb{P}^{1}(\mathbb{R})$. This defines a closed geodesic in the quotient $X_{\Gamma}$ of length $\log \epsilon$, for $\epsilon>1$ the generator of $V=U_{L}^{+}=\epsilon^{\mathbb{Z}}$. We denote the closed geodesic by $\bar{\gamma}_{\theta, \theta^{\prime}}$.

Consider the restriction of the universal family $\mathcal{U}_{\Gamma}$ to the closed geodesic $\bar{\gamma}_{\theta, \theta^{\prime}}$. Via the parameterization of the closed geodesic by a circle $S^{1}$ of length $\log \epsilon$, we can consider the pullback to the parameterizing $S^{1}$ of $\mathcal{U}_{\Gamma}$. We obtain in this way a real 3 -dimensional manifold, which we denote $\mathcal{U}_{\theta, \theta^{\prime}}$. This is topologically a 3-manifold that fibers over a circle, with fibers $T^{2}$. We consider it endowed with the metric that is the product of the geodesic length and the flat metric on $T^{2}$. We then obtain the following result.

Lemma 2.4. The solvmanifold $S(\Lambda, V)$ is isometrically equivalent to $\mathcal{U}_{\theta, \theta^{\prime}}$.

Proof. We recall the following procedure of Hecke to lift closed geodesics to the space of lattices [18, Section 1.8.2]. Given a lattice $L$ in a real quadratic 
field $\mathbb{K}$, with $\ell \mapsto \ell^{\prime}$ the Galois involution, one sets

$$
\Lambda_{t}(L):=\left\{z \in \mathbb{H} \mid z=z(\ell, t)=\ell e^{t}+i \ell^{\prime} e^{-t} \ell \in L\right\}
$$

This defines, for all $t \in \mathbb{R}$ a lattice $\Lambda_{t} \subset \mathbb{C}$. The action of $V=\epsilon^{\mathbb{Z}}$ is of the form (cf. [18, Lemma 1.8.3])

$$
z(\ell, t) \longmapsto \epsilon \ell e^{t}+i \epsilon^{\prime} \ell^{\prime} e^{-t}=z(\ell, t+\log \epsilon)
$$

In particular (see again [18, Lemma 1.8.3]), for $\{1, \theta\}$ a basis of $L$, the lattice $\Lambda_{t}(L)$ is generated by $\left\{1, \tau_{t}\right\}$ where $\tau_{t}$ runs over the geodesic $\ell_{\theta, \theta^{\prime}} \subset \mathbb{H}$, for $t \in \mathbb{R}$. Thus, we can identify the 3 -manifold $\mathcal{U}_{\theta, \theta^{\prime}}$ with the fibration over a circle of length $\log \epsilon$, with fiber $E_{\tau_{t}}=\mathbb{C} / \Lambda_{t}(L)$.

On the other hand, the 3 -manifold $S(\Lambda, V)$ is a fibration of tori over the circle

$$
T^{2} \longrightarrow S(\Lambda, V) \longrightarrow S^{1}
$$

where the base $S^{1}$ is a circle of length $\log \epsilon$ and the fiber over $t \in S^{1}$ is given by the 2-torus

$$
T_{t}^{2}=\mathbb{R}^{2} / \Lambda_{t}
$$

with $\Lambda_{t}=\Theta_{t}(\Lambda)$, for $\Theta_{t}(x, y)=\left(e^{t} x, e^{-t} y\right)$. This proves the result.

\section{Actions on noncommutative tori with real multiplication}

The noncommutative torus $\mathcal{A}_{\theta}$ of modulus $\theta \in \mathbb{R} \backslash \mathbb{Q}$ is the noncommutative space described, at the topological level, by the irrational rotation $C^{*}$-algebra, that is, the universal $C^{*}$-algebra generated by two unitaries $U$, $V$ with the commutation relation $V U=e^{2 \pi i \theta} U V$. It has a smooth structure given by the smooth subalgebra of series $\sum_{n, m} a_{n, m} U^{n} V^{m}$ with rapidly decaying coefficients [9].

It is a well-known result $[9,27]$ that the algebras $\mathcal{A}_{\theta_{1}}$ and $\mathcal{A}_{\theta_{2}}$ are Morita equivalent whenever there exists an element $g \in \mathrm{SL}_{2}(\mathbb{Z})$ acting on $\mathbb{R}$ by fractional linear transformations, such that $\theta_{1}=g \theta_{2}$. In the following, we concentrate on the case where the irrational number $\theta$ is a quadratic irrationality in a real quadratic field $\mathbb{K}=\mathbb{Q}(\theta)$. These are the noncommutative tori with real multiplication considered in [18]. We let $L$ be the lattice in $\mathbb{K}$ with $\iota_{1}(L)=\mathbb{Z}+\mathbb{Z} \theta$ and $\iota_{2}(L)=\mathbb{Z}+\mathbb{Z} \theta^{\prime}$. As before, we denote by $\Lambda$ the corresponding lattice in $\mathbb{R}^{2}$. 
The $C^{*}$-algebra of the noncommutative torus $\mathcal{A}_{\theta}$ described above can be equivalently described as the crossed product

$$
\mathcal{A}_{\theta}=C\left(S^{1}\right) \rtimes_{\theta} \mathbb{Z},
$$

where the action of $\mathbb{Z}$ on $S^{1}$ is by the irrational rotation by $\exp (2 \pi i \theta)$. Up to Morita equivalence, one can replace $C\left(S^{1}\right)$ by the crossed product $C_{0}(\mathbb{R}) \rtimes$ $\mathbb{Z}$, and one obtains a Morita equivalent description of the noncommutative torus as

$$
C_{0}(\mathbb{R}) \rtimes_{\theta} \mathbb{Z}^{2}=C_{0}(\mathbb{R}) \rtimes(\mathbb{Z}+\mathbb{Z} \theta) .
$$

In the case we are considering, of real quadratic fields, we can regard the noncommutative torus with real multiplication associated to a lattice $L \subset \mathbb{K}$ as described by the algebras

$$
\mathbb{T}_{\Lambda, 1}:=C_{0}(\mathbb{R}) \rtimes \iota_{1}(L), \quad \mathbb{T}_{\Lambda, 2}:=C_{0}(\mathbb{R}) \rtimes \iota_{2}(L)
$$

These algebras can be described as follows. They are $C^{*}$-algebras generated by elements of the form $f U_{\lambda}$, with $f \in C_{0}(\mathbb{R})$ and $\lambda \in \Lambda$, with the product

$$
f U_{\lambda} h U_{\eta}=f U_{\lambda, i}(h) U_{\lambda+\eta}, \quad \text { where } U_{\lambda, i}(h)(x)=h\left(x+\iota_{i}(\lambda)\right), \quad i=1,2 .
$$

The group $V=\epsilon^{\mathbb{Z}}$ of units acts as symmetries of the noncommutative tori $\mathbb{T}_{\Lambda, i}$ as follows.

Lemma 3.1. For $k \in \mathbb{Z}$ and $f U_{\lambda} \in \mathbb{T}_{\Lambda, i}$, set $v_{\epsilon}^{k}(f)(x):=f\left(\epsilon^{k} x\right)$ and

$$
v_{1}^{k}\left(f U_{\lambda}\right)=v_{\epsilon}^{k}(f) U_{A_{\epsilon}^{k}(\lambda)} \quad \text { and } \quad v_{2}^{k}\left(f U_{\lambda}\right)=v_{\epsilon^{\prime}}^{k}(f) U_{A_{\epsilon}^{k}(\lambda)} .
$$

This defines actions $v_{i}: V \rightarrow \operatorname{Aut}\left(\mathbb{T}_{\Lambda, i}\right)$.

Proof. The result follows directly from

$$
v_{i}^{k}\left(U_{\lambda}(h)\right)(x)=U_{A_{\epsilon}^{k}(\lambda)}\left(v_{i}^{k}(h)\right)(x)= \begin{cases}h\left(\epsilon^{k}(x+n+m \theta)\right), & i=1, \\ h\left(\epsilon^{-k}\left(x+n+m \theta^{\prime}\right)\right), & i=2\end{cases}
$$

which implies that

$$
v_{\epsilon}^{k}\left(f U_{\lambda} h U_{\eta}\right)=v_{i}^{k}(f) v_{i}^{k}\left(U_{\lambda}(h)\right) U_{A_{\epsilon}^{k}(\lambda+\eta)}=v_{i}^{k}\left(f U_{\lambda}\right) v_{i}^{k}\left(h U_{\eta}\right) .
$$


It is customary, in noncommutative geometry, to replace quotients by crossed product algebras. In this case, the quotient of the noncommutative tori $\mathbb{T}_{\Lambda, i}$ by the action of $V$ is described by the crossed product algebra

$$
\mathbb{T}_{\Lambda, V, i}:=\mathbb{T}_{\Lambda, i} \rtimes_{v_{i}} V
$$

which we can view equivalently as the crossed product

$$
\mathbb{T}_{\Lambda, V, i}:=C_{0}(\mathbb{R}) \rtimes_{i} S(\Lambda, V)
$$

for the actions of $S(\Lambda, V)$ on $C_{0}(\mathbb{R})$ of the form

$$
U_{(\lambda, k)} f(x)=f\left(\epsilon^{k}(x+n+m \theta)\right) \quad \text { or } \quad U_{(\lambda, k)} f(x)=f\left(\epsilon^{-k}\left(x+n+m \theta^{\prime}\right)\right) .
$$

\section{Twisted group algebras and the magnetic Laplacian}

Another equivalent description of the algebra $\mathcal{A}_{\theta}$ of the noncommutative torus is as twisted group $C^{*}$-algebra. This played an important role in the context of the noncommutative geometry model of the integer quantum Hall effect (see [5]).

We recall briefly the definition and properties of twisted group $C^{*}$ algebras, as this will be useful in the following. For a similar overview and applications to the case of Fuchsian groups, see [21].

\subsection{Twisted group algebras}

Let $\Gamma$ be a finitely generated discrete group, and let $\sigma: \Gamma \times \Gamma \rightarrow U(1)$ be a multiplier, that is, a 2-cocycle satisfying the cocycle property

$$
\sigma\left(\gamma_{1}, \gamma_{2}\right) \sigma\left(\gamma_{1} \gamma_{2}, \gamma_{3}\right)=\sigma\left(\gamma_{1}, \gamma_{2} \gamma_{3}\right) \sigma\left(\gamma_{2}, \gamma_{3}\right)
$$

with $\sigma(\gamma, 1)=\sigma(1, \gamma)=1$.

Consider then the Hilbert space $\ell^{2}(\Gamma)$ and the left/right $\sigma$-regular representations of $\Gamma$ given by

$$
L_{\gamma}^{\sigma} f\left(\gamma^{\prime}\right)=f\left(\gamma^{-1} \gamma^{\prime}\right) \sigma\left(\gamma, \gamma^{-1} \gamma^{\prime}\right), \quad R_{\gamma}^{\sigma} f\left(\gamma^{\prime}\right)=f\left(\gamma^{\prime} \gamma\right) \sigma\left(\gamma^{\prime}, \gamma\right)
$$

They satisfy the relations

$$
L_{\gamma}^{\sigma} L_{\gamma^{\prime}}^{\sigma}=\sigma\left(\gamma, \gamma^{\prime}\right) L_{\gamma \gamma^{\prime}}^{\sigma}, \quad R_{\gamma}^{\sigma} R_{\gamma^{\prime}}^{\sigma}=\sigma\left(\gamma, \gamma^{\prime}\right) R_{\gamma \gamma^{\prime}}^{\sigma}
$$


Moreover, the left $\sigma$-regular representation commutes with the right $\bar{\sigma}$-regular representation, with $\bar{\sigma}$ the conjugate multiplier. The algebra generated by the $R_{\gamma}^{\sigma}$ is the twisted group ring $\mathbb{C}(\Gamma, \sigma)$. Its norm closure is the (reduced) twisted group $C^{*}$-algebra $C_{r}^{*}(\Gamma, \sigma)$.

\subsection{The noncommutative tori as twisted group algebras}

One identifies the $C^{*}$-algebra $\mathcal{A}_{\theta}$ of the noncommutative torus with the reduced twisted group $C^{*}$-algebra $C_{r}^{*}\left(\mathbb{Z}^{2}, \sigma\right)$ in the following way. Let $\sigma$ be a cocycle of the form

$$
\sigma\left((n, m),\left(n^{\prime}, m^{\prime}\right)\right):=\exp \left(-2 \pi i\left(\xi_{1} n m^{\prime}+\xi_{2} m n^{\prime}\right)\right)
$$

Then the operators $U=R_{(0,1)}^{\sigma}$ and $V=R_{(1,0)}^{\sigma}$ acting by

$$
U f(n, m)=e^{-2 \pi i \xi_{2} n} f(n, m+1), \quad V f(n, m)=e^{-2 \pi i \xi_{1} m} f(n+1, m)
$$

which generate the algebra $C_{r}^{*}\left(\mathbb{Z}^{2}, \sigma\right)$ satisfy the commutation relation

$$
U V=e^{2 \pi i \theta} V U, \quad \text { with } \theta=\xi_{2}-\xi_{1}
$$

Notice that different choices of $\xi_{1}, \xi_{2}$ with the same $\theta=\xi_{2}-\xi_{1}$ yield the same algebra $\mathcal{A}_{\theta}$. This gives us the freedom to choose the $\xi_{i}$ according to the following result.

Lemma 4.1. A cocycle $\sigma$ of the form (4.4) has the property that

$$
\sigma\left((n, m),\left(n^{\prime}, m^{\prime}\right)\right)=\sigma\left((n, m) \varphi,\left(n^{\prime}, m^{\prime}\right) \varphi\right), \quad \forall \varphi=\left(\begin{array}{ll}
a & b \\
c & d
\end{array}\right) \in \operatorname{SL}_{2}(\mathbb{Z})
$$

if and only if $\xi_{2}=-\xi_{1}$.

Proof. We see that $\sigma\left((n, m) \varphi,\left(n^{\prime}, m^{\prime}\right) \varphi\right)$ is of the form

$$
\begin{aligned}
& \exp \left(-2 \pi i\left(\left(\xi_{1}+\xi_{2}\right)\left(a b n n^{\prime}+c d m m^{\prime}\right)+\left(\xi_{1} c b+\xi_{2} a d\right) m n^{\prime}\right.\right. \\
& \left.\left.\quad+\left(\xi_{1} a d+\xi_{2} c b\right) n m^{\prime}\right)\right)
\end{aligned}
$$


Thus, in the following we will assume that $\xi_{2}=\theta / 2=-\xi_{1}$ in the choice of the cocycle $\sigma$ of (4.4). We can then write $\sigma$ in the form

$$
\sigma_{\theta}((n, m),(k, r))=\exp (\pi i \theta(n r-m k))=\exp (\pi i \theta(n, m) \wedge(k, r)),
$$

where we use the notation

$$
(a, b) \wedge(c, d)=\operatorname{det}\left(\begin{array}{ll}
a & b \\
c & d
\end{array}\right) .
$$

We then obtain the following identifications.

Corollary 4.2. The noncommutative tori $\mathbb{T}_{\Lambda, i}$ are described by twisted group $C^{*}$-algebras

$$
\begin{aligned}
& \mathbb{T}_{\Lambda, 1}=C^{*}\left(\mathbb{Z}^{2}, \sigma_{\theta}\right)=C^{*}\left(\Lambda, \sigma_{\theta\left(\theta^{\prime}-\theta\right)^{-1}}\right) \\
& \mathbb{T}_{\Lambda, 2}=C^{*}\left(\mathbb{Z}^{2}, \sigma_{\theta^{\prime}}\right)=C^{*}\left(\Lambda, \sigma_{\theta^{\prime}\left(\theta^{\prime}-\theta\right)^{-1}}\right) .
\end{aligned}
$$

Proof. The expression

$$
\sigma_{u}(\lambda, \eta)=\exp (\pi i u \lambda \wedge \eta)
$$

defines a cocycle on $\Lambda$. For $\lambda=\left(n+m \theta, n+m \theta^{\prime}\right)$ and $\eta=\left(k+r \theta, k+r \theta^{\prime}\right)$, a direct calculation shows that

$$
\sigma_{\theta}((n, m),(k, r))=\sigma_{u}(\lambda, \eta), \quad \text { for } \quad u=\theta\left(\theta^{\prime}-\theta\right)^{-1} .
$$

Thus, the generators $R_{(n, m)}^{\sigma}$ of $C^{*}\left(\mathbb{Z}^{2}, \sigma_{\theta}\right)$ with

$$
R_{(n, m)}^{\sigma} R_{(k, r)}^{\sigma}=\sigma_{\theta}((n, m),(k, r)) R_{(n, m)+(k, r)}^{\sigma}
$$

are identified with the generators $R_{\lambda}^{\sigma}$ of $C^{*}\left(\Lambda, \sigma_{\theta\left(\theta^{\prime}-\theta\right)^{-1}}\right)$ with

$$
R_{\lambda}^{\sigma} R_{\eta}^{\sigma}=\sigma_{\theta\left(\theta^{\prime}-\theta\right)^{-1}}(\lambda, \eta) R_{\lambda+\eta}^{\sigma}
$$

The case of $\mathbb{T}_{\Lambda, 2}$ is analogous.

\subsection{Twisted group algebra of $S(\Lambda, V)$}

We now show that the algebra $\mathcal{A}_{\theta} \rtimes V$, which we introduced in the previous section to describe the quotient of the noncommutative torus with real multiplication by the action of $V$, also admits a description in terms 
of twisted group $C^{*}$-algebras, for the group $S(\Lambda, V)$. First notice that the group $S(\Lambda, V)$ is amenable, so that the maximal and reduced group $C^{*}$ algebras coincide, $C_{\max }^{*}(S(\Lambda, V)) \cong C_{r}^{*}(S(\Lambda, V))$, so that we can simply write $C^{*}(S(\Lambda, V))$ and $C^{*}(S(\Lambda, V), \tilde{\sigma})$ for the twisted case.

Lemma 4.3. Let $\sigma$ be a multiplier on $\mathbb{Z}^{2}$ of the form (4.4), with $\xi_{2}=\theta / 2=$ $-\xi_{1}$. Then the map $\tilde{\sigma}: S(\Lambda, V) \times S(\Lambda, V) \rightarrow U(1)$ of the form

$$
\tilde{\sigma}\left((n, m, k),\left(n^{\prime}, m^{\prime}, k^{\prime}\right)\right):=\sigma\left((n, m),\left(n^{\prime}, m^{\prime}\right) \varphi_{\epsilon}^{k}\right)
$$

is a multiplier for $S(\Lambda, V)$, identified with the group $\mathbb{Z}^{2} \rtimes_{\varphi_{\epsilon}} \mathbb{Z}$.

Proof. The cocycle condition for $\sigma$ and the $\mathrm{SL}_{2}(\mathbb{Z})$-invariance $\sigma((n, m) \varphi$, $\left.\left(n^{\prime}, m^{\prime}\right) \varphi\right)=\sigma\left((n, m),\left(n^{\prime} m^{\prime}\right)\right)$ imply that $\tilde{\sigma}$ also satisfies the cocycle condition (4.1), since we have

$$
\begin{aligned}
& \sigma(\left.\left(n_{1}, m_{1}\right),\left(n_{2}, m_{2}\right) \varphi_{\epsilon}^{k_{1}}\right) \sigma\left(\left(n_{1}, m_{1}\right)+\left(n_{2}, m_{2}\right) \varphi_{\epsilon}^{k_{1}},\left(n_{3}, m_{3}\right) \varphi_{\epsilon}^{k_{1}+k_{2}}\right) \\
&= \sigma\left(\left(n_{1}, m_{1}\right),\left(n_{2}, m_{2}\right) \varphi_{\epsilon}^{k_{1}}+\left(n_{3}, m_{3}\right) \varphi_{\epsilon}^{k_{1}+k_{2}}\right) \\
& \quad \times \sigma\left(\left(n_{2}, m_{2}\right) \varphi_{\epsilon}^{k_{1}},\left(n_{3}, m_{3}\right) \varphi_{\epsilon}^{k_{1}+k_{2}}\right) .
\end{aligned}
$$

We then have the following result.

Proposition 4.4. The algebras $\mathbb{T}_{\Lambda, V, i}=\mathbb{T}_{\Lambda, i} \rtimes_{i} V$ are isomorphic to the algebras

$$
\begin{aligned}
\mathbb{T}_{\Lambda, V, 1} & =C^{*}\left(\mathbb{Z}^{2}, \sigma_{\theta}\right) \rtimes_{v_{1}} \mathbb{Z}=C^{*}\left(\mathbb{Z}^{2} \rtimes_{\varphi_{\epsilon}} \mathbb{Z}, \tilde{\sigma}_{\theta}\right) \\
& =C^{*}\left(\Lambda, \sigma_{\theta\left(\theta^{\prime}-\theta\right)^{-1}}\right) \rtimes_{v_{1}} V=C^{*}\left(S(\Lambda, V), \tilde{\sigma}_{\theta\left(\theta^{\prime}-\theta\right)^{-1}}\right), \\
\mathbb{T}_{\Lambda, V, 2} & =C^{*}\left(\mathbb{Z}^{2}, \sigma_{\theta^{\prime}}\right) \rtimes_{v_{2}} \mathbb{Z}=C^{*}\left(\mathbb{Z}^{2} \rtimes_{\varphi_{\epsilon}} \mathbb{Z}, \tilde{\sigma}_{\theta^{\prime}}\right) \\
& =C^{*}\left(\Lambda, \sigma_{\theta^{\prime}\left(\theta^{\prime}-\theta\right)^{-1}}\right) \rtimes_{v_{2}} V=C^{*}\left(S(\Lambda, V), \tilde{\sigma}_{\theta^{\prime}\left(\theta^{\prime}-\theta\right)^{-1}}\right) .
\end{aligned}
$$

Proof. We just show explicitly one of the identifications. The others follow similarly. The twisted group algebra $C^{*}\left(\mathbb{Z}^{2} \rtimes_{\varphi_{\epsilon}} \mathbb{Z}, \tilde{\sigma}_{\theta}\right)$ is generated by elements $R_{(n, m, k)}^{\tilde{\sigma}}$ satisfying

$$
R_{(n, m, k)}^{\tilde{\sigma}} R_{\left(n^{\prime}, m^{\prime}, k^{\prime}\right)}^{\tilde{\sigma}}=\tilde{\sigma}\left((n, m, k),\left(n^{\prime}, m^{\prime}, k^{\prime}\right)\right) R_{(n, m, k)\left(n^{\prime}, m^{\prime}, k^{\prime}\right)}^{\tilde{\sigma}}
$$


The crossed product $C^{*}\left(\mathbb{Z}^{2}, \sigma_{\theta}\right) \rtimes_{v_{1}} \mathbb{Z}$ is generated by elements of the form $R_{(n, m)}^{\sigma} v_{\epsilon}^{k}$. The map $R_{(n, m, k)}^{\tilde{\sigma}} \mapsto R_{(n, m)}^{\sigma} v_{\epsilon}^{k}$ gives an identification of the generators, which also satisfies

$$
\begin{aligned}
R_{(n, m)}^{\sigma} v_{\epsilon}^{k} R_{\left(n^{\prime}, m^{\prime}\right)}^{\sigma} v_{\epsilon}^{k^{\prime}} & =R_{(n, m)}^{\sigma} R_{\left(n^{\prime}, m^{\prime}\right) \varphi_{\epsilon}^{k}}^{\sigma} v_{\epsilon}^{k+k^{\prime}} \\
& =\sigma\left((n, m),\left(n^{\prime}, m^{\prime}\right) \varphi_{\epsilon}^{k}\right) R_{(n, m)+\left(n^{\prime}, m^{\prime}\right) \varphi_{\epsilon}^{k}}^{\sigma} v_{\epsilon}^{k+k^{\prime}} .
\end{aligned}
$$

This gives an isomorphism $C^{*}\left(\mathbb{Z}^{2}, \sigma_{\theta}\right) \rtimes_{v_{1}} \mathbb{Z}=C^{*}\left(\mathbb{Z}^{2} \rtimes_{\varphi_{\epsilon}} \mathbb{Z}, \tilde{\sigma}_{\theta}\right)$.

\subsection{The magnetic Laplacian}

Consider the general setting of a finitely generated discrete group $\Gamma$ acting freely on a contractible space $\tilde{X}$ with compact quotient $X=\tilde{X} / \Gamma$. Assume everything happens in the smooth category and we think of $\tilde{X}$ as endowed with a metric that is invariant under the action of $\Gamma$. Upon choosing a base point $x_{0} \in \tilde{X}$, we can think of the discrete set $\Gamma x_{0}$ as a crystal of charged ions and consider the electron-ion interaction problem in $\tilde{X}$. This means that electrons move in $\tilde{X}$ subject to a periodic potential. Under reasonable assumptions, one can make an independent electron approximation and replace the $N$-particle Hamiltonian with the unbounded periodic electric potential of the ion crystal with a single electron Hamiltonian in an effective periodic potential given by a bounded function (see [21] for a brief overview).

The Hamiltonian is then of the form $\Delta+V$, where the $\Delta$ is the Laplacian on $\tilde{X}$. We think of it as an unbounded operator on $L^{2}(\tilde{X})$. The Hamiltonian is invariant under translations by $\gamma \in \Gamma$, that is, $T_{\gamma} \Delta=\Delta T_{\gamma}$ and by construction $V$ is also invariant. Here the $T_{\gamma}$ are the operators $T_{\gamma} f(x)=f(x \gamma)$ on $L^{2}(\tilde{X})$.

One can consider on $\tilde{X}$ a magnetic field. This is specified by a closed 2 -form $\omega$ which satisfies $\gamma^{*} \omega=\omega$. Since $\tilde{X}$ is contractible, there is a global magnetic potential $\omega=d \chi$. The corresponding hermitian connection $\nabla=$ $d-i \chi$ satisfies $\nabla^{2}=i \omega$. The invariance of $\omega$ implies $d\left(\chi-\gamma^{*} \chi\right)=0$, so that $\chi-\gamma^{*} \chi=d \phi_{\gamma}$, where the function $\phi_{\gamma}(x)=\int_{x_{0}}^{x} \chi-\gamma^{*} \chi$ has the properties that

$$
\phi_{\gamma}(x)-\phi_{\gamma^{\prime}}(\gamma x)-\phi_{\gamma \gamma^{\prime}}(x)
$$

is independent of $x \in \tilde{X}$ and $\phi_{\gamma}\left(x_{0}\right)=0$, so that

$$
\sigma\left(\gamma, \gamma^{\prime}\right)=\exp \left(-i \phi_{\gamma}\left(\gamma^{\prime} x_{0}\right)\right)
$$

defines a multiplier $\sigma: \Gamma \times \Gamma \rightarrow U(1)$. The Laplacian $\Delta$ is naturally replaced, in the presence of a magnetic field, by the magnetic Laplacian $\Delta^{\chi}=\nabla^{*} \nabla=$ 
$(d-i \chi)^{*}(d-i \chi)$. This is no longer invariant under translations $T_{\gamma}$, but is invariant under the magnetic translations

$$
T_{\gamma}^{\phi} \Delta^{\chi}=\Delta^{\chi} T_{\gamma}^{\phi}
$$

where $T_{\gamma}^{\phi} f(x)=\exp \left(i \phi_{\gamma}(x)\right) f\left(\gamma^{-1} x\right)$. Similarly, in the independent electron approximation, the effective potential $V$ is also invariant under the magnetic translations.

The magnetic translations satisfy the relations of the twisted group algebra $C_{r}^{*}(\Gamma, \bar{\sigma})$

$$
T_{\gamma}^{\phi} T_{\gamma^{\prime}}^{\phi}=\bar{\sigma}\left(\gamma, \gamma^{\prime}\right) T_{\gamma \gamma^{\prime}}^{\phi}
$$

for $\sigma$ as in (4.10) and $\bar{\sigma}$ the conjugate. (We refer the reader to [19,21] for a brief overview of these well-known facts.)

\subsection{Discretized electron-ion interaction and Harper operators}

It is usually convenient to discretize the electron-ion interaction problem. This means replacing the continuum model with Hilbert space $L^{2}(\tilde{X})$ by a discrete model on the Hilbert space $\ell^{2}(\Gamma)$. In the case without magnetic field, this is done by replacing the Laplacian $\Delta$ by its discretized version $\Delta_{\text {discr }}=r-\mathcal{R}$, where $r$ is the cardinality of a symmetric set of generators for $\Gamma$ and $\mathcal{R}$ is the random walk operator

$$
\mathcal{R}=\sum_{i=1}^{r} R_{\gamma_{i}} \quad \text { with } \quad R_{\gamma_{i}} f(\gamma)=f\left(\gamma \gamma_{i}\right)
$$

for $f \in \ell^{2}(\Gamma)$. As in the continuum model, the discretized Laplacian commutes with translations by elements $\gamma \in \Gamma$. The effective potential is then taken to be an element in the group ring $\mathbb{C}[\Gamma]$.

In the presence of a magnetic field, one can still obtain a good discretized version of the electron-ion interaction problem as in [30]. The random walk operator of (4.12) is then replaced by the Harper operator

$$
\mathcal{H}_{\sigma}=\sum_{i=1}^{r} R_{\gamma_{i}}^{\sigma}
$$

with $R_{\gamma_{i}}^{\sigma} \in \mathbb{C}(\Gamma, \sigma)$ the elements of the right $\sigma$-regular representation, with $\sigma$ the cocycle of (4.10). The discretized version of the magnetic Laplacian 
is then given by the operator

$$
\Delta_{\text {discr }}^{\chi}=r-\mathcal{H}_{\sigma},
$$

which commutes with the magnetic translations $L_{\gamma}^{\bar{\sigma}}$. Similarly, the effective potential is taken to be an element $V \in \mathbb{C}(\Gamma, \sigma)$, which then also commutes with the magnetic translations $L_{\gamma}^{\bar{\sigma}}$.

\subsection{Harper operators for noncommutative tori and for $S(\Lambda, V)$}

In the case of the noncommutative torus, viewed as the twisted group $C^{*}$ algebra $C^{*}\left(\mathbb{Z}^{2}, \sigma\right)$, the Harper operator is of the form

$$
\mathcal{H}_{\sigma}=U+U^{*}+V+V^{*},
$$

where $U$ and $V$ are the generators of $\mathcal{A}_{\theta}$.

The spectral theory of the Harper operator $\mathcal{H}_{\sigma}$ of (4.15) was widely studied. In particular, it was shown in [17] that the spectrum exhibits a remarkable fractal structure (the Hofstadter butterfly) which appears to have infinitely many gaps (Cantor like spectrum) for irrational $\theta$ and finitely many gaps (band spectrum) for rational $\theta$. The precise gap structure of the spectrum of Harper operators, as a function of the magnetic flux (that is $\theta$ in the noncommutative torus case), is a problem still under active investigation. As we see more in detail in the following, in the specific case of interest here, the gap labelling problem for the spectrum of the Harper operator is closely related to the computation of the range of the trace on the $K$-theory of the twisted group $C^{*}$-algebra.

In the following, we will be interested in the case of the group $S(\Lambda, V)$. In this case, after identifying it with $\mathbb{Z}^{2} \rtimes_{\varphi_{\epsilon}} \mathbb{Z}$, the Harper operator is of the form

$$
\mathcal{H}_{\tilde{\sigma}}=U+U^{*}+V+V^{*}+W+W^{*},
$$

where $U=R_{(0,1,0)}^{\tilde{\sigma}}, V=R_{(1,0,0)}^{\tilde{\sigma}}$ and $W=R_{(0,0,1)}^{\tilde{\sigma}}$.

\subsection{Spectral theory and $K$-theory}

We recall here briefly the relation between spectral theory of Harper operators and $K$-theory of twisted group $C^{*}$-algebras (cf. [6; 21, Section 3]). We then proceed in the following section to analyze the specific case of $C^{*}(S(\Lambda, V))$. 
As we have seen, the twisted group $C^{*}$-algebra $C_{r}^{*}(\Gamma, \sigma)$ is the norm closure of the twisted group ring $\mathbb{C}(\Gamma, \sigma)$ in the right $\sigma$-regular representation on $\ell^{2}(\Gamma)$, that is, the $C^{*}$-algebra generated by the magnetic translations $R_{\gamma}^{\sigma}$. If we take the weak closure of $\mathbb{C}(\Gamma, \sigma)$, we obtain the twisted group von Neumann algebra $\mathcal{U}(\Gamma, \sigma)$. Suppose given an operator $\mathcal{H}_{\sigma, V}=\mathcal{H}_{\sigma}+V$, with $\mathcal{H}_{\sigma}$ the Harper operator described above and $V$ an effective potential in $\mathbb{C}(\Gamma, \sigma)$. We have by construction $\mathcal{H}_{\sigma, V} \in \mathbb{C}(\Gamma, \sigma) \subset C_{r}^{*}(\Gamma, \sigma) \subset \mathcal{U}(\Gamma, \sigma)$, hence the spectral projections of $\mathcal{H}_{\sigma, V}$,

$$
P_{E}=\mathbf{1}_{(-\infty, E]}\left(\mathcal{H}_{\sigma, V}\right)
$$

are in the von Neumann algebra, $P_{E} \in \mathcal{U}(\Gamma, \sigma)$. In particular, if the energy level $E$ is not in the spectrum of $\mathcal{H}_{\sigma, V}$, then the corresponding spectral projection $P_{E}$ is actually in the $C^{*}$-algebra $C_{r}^{*}(\Gamma, \sigma)$.

This implies that the question of counting gaps in the spectrum of $\mathcal{H}_{\sigma, V}$ can be reformulated as a problem of counting projections in the $C^{*}$-algebra $C_{r}^{*}(\Gamma, \sigma)$, modulo the Murray-von Neumann equivalence relation, $P \sim Q$ if there exists $V \in C_{r}^{*}(\Gamma, \sigma) \otimes \mathcal{K}$ with $P=V^{*} V$ and $Q=V V^{*}$. Equivalent spectral projections correspond to a same gap in the spectrum. The group $K_{0}\left(C_{r}^{*}(\Gamma, \sigma)\right)$ is the Grothendieck group of the resulting abelian semi-group (with the operation of direct sum). Thus, the gap counting problem is restated as a problem involving $K$-theory of $C^{*}$-algebras. More precisely, there is a faithful canonical finite trace

$$
\tau(a)=\left\langle a \delta_{1}, \delta_{1}\right\rangle_{\ell^{2}(\Gamma)},
$$

on $C_{r}^{*}(\Gamma, \sigma)$, with $\delta_{\gamma}$ the canonical basis of $\ell^{2}(\Gamma)$. This extends to

$$
\left.\operatorname{tr}=\tau \otimes \operatorname{Tr}:\left\{P \in C_{r}^{*}(\Gamma, \sigma) \otimes \mathcal{K}\right) \mid P^{*}=P, P^{2}=P\right\} \longrightarrow \mathbb{R},
$$

with $\operatorname{Tr}$ the standard trace on bounded operators and induces

$$
[\operatorname{tr}]: K_{0}\left(C_{r}^{*}(\Gamma, \sigma)\right) \longrightarrow \mathbb{R}
$$

One can obtain an estimate of the number of equivalence classes of projections by a direct computation of the range of the trace on $\left.K_{0}\left(C_{r}^{*}(\Gamma, \sigma)\right)\right)$, using

$$
\left.\operatorname{tr}\left(\left\{P \in C_{r}^{*}(\Gamma, \sigma) \otimes \mathcal{K}\right) \mid P^{*}=P, P^{2}=P\right\}\right)=[\operatorname{tr}]\left(K_{0}\left(C_{r}^{*}(\Gamma, \sigma)\right)\right) \cap[0,1] .
$$




\section{Homotopy quotient and the Baum-Connes conjecture}

As we show in this section, the computation of the range of the trace on $K$-theory is closely related to the use of the 3 -manifold $X_{\epsilon}$ as a commutative model up to homotopy of the noncommutative space $\mathbb{T}_{\Lambda, V, i}$.

The main idea of the Baum-Connes conjecture is precisely the fact that noncommutative spaces originating from "bad quotients" have good homotopy quotients that can be used to compute geometrically invariants such as the analytic K-theory.

The group $S(\Lambda, V)$ we are considering here is a particular case of a class of groups of the form $\mathbb{Z}^{2} \rtimes_{\varphi} \mathbb{Z}$, for some $\varphi \in \mathrm{SL}_{2}(\mathbb{Z})$. The corresponding (twisted) group $C^{*}$-algebras and their $K$-theory were analyzed in [25]. We wish to stress here the relation between the noncommutative space and its model $X_{\epsilon}$ and the role of the latter in the index computations.

\section{1. $K$-theory of $C^{*}(S(\Lambda, V), \tilde{\sigma})$}

We now compute explicitly the $K$-theory of the twisted group $C^{*}$-algebra of $S(\Lambda, V)$. This can be done using the Pimsner-Voiculescu six terms exact sequence.

Lemma 5.1. The K-theory groups of $\left.C^{*}(S(\Lambda, V), \tilde{\sigma})\right)$ are of the form

$$
\begin{aligned}
& K_{0}\left(C^{*}(S(\Lambda, V), \tilde{\sigma})\right) \cong \Lambda, \\
& K_{1}\left(C^{*}(S(\Lambda, V), \tilde{\sigma})\right) \cong \Lambda \oplus \Lambda /\left(1-A_{\epsilon}\right) \Lambda .
\end{aligned}
$$

Proof. By Proposition 4.4, we can identify $C^{*}(S(\Lambda, V), \tilde{\sigma})$ with the crossed product $C^{*}(\Lambda, \sigma) \rtimes V$. Thus, we can apply the Pimsner-Voiculescu six terms exact sequence for the actions of $V \cong \mathbb{Z}$. We have

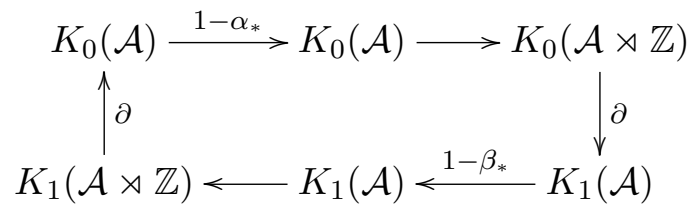

where $\mathcal{A}=C^{*}(\Lambda, \sigma)$ and $\alpha_{*}$ and $\beta_{*}$ denote the action on $K_{0}(\mathcal{A})$ and $K_{1}(\mathcal{A})$, respectively, induced by the generator $A_{\epsilon}$ of the $\mathbb{Z}$-action on $\mathcal{A}$. We can identify $K_{0}(\mathcal{A})=\Lambda=K_{1}(\mathcal{A})$. We then have $1-\alpha_{*}=0$ and $1-\beta_{*}=1-$ $A_{\epsilon}$, so that we obtain $\operatorname{Ker}\left(1-\beta_{*}\right)=\operatorname{Ker}\left(1-A_{\epsilon}\right)=0$ and $\operatorname{Coker}\left(1-\beta_{*}\right)=$ $\Lambda /\left(1-A_{\epsilon}\right) \Lambda$. 
We find in this way an abstract isomorphism of abelian groups

$$
\begin{aligned}
& K_{0}\left(C^{*}(S(\Lambda, V)), \tilde{\sigma}\right) \cong H^{\text {odd }}\left(X_{\epsilon}, \mathbb{Z}\right) \cong \mathbb{Z}^{2} \\
& K_{1}\left(C^{*}(S(\Lambda, V)), \tilde{\sigma}\right) \cong H^{\mathrm{ev}}\left(X_{\epsilon}, \mathbb{Z}\right) \cong \mathbb{Z}^{2} \oplus \operatorname{Coker}\left(1-A_{\epsilon}\right)
\end{aligned}
$$

This identification can be justified more naturally in terms of the BaumConnes conjecture, as we discuss in the following.

\section{2. $K$-theory and the twist}

The following result shows that the presence of the twisting by $\tilde{\sigma}$ has no effect on the $K$-theory.

Lemma 5.2. There is an isomorphism

$$
K_{i}\left(C^{*}(S(\Lambda, V), \tilde{\sigma})\right) \cong K_{i}\left(C^{*}(S(\Lambda, V))\right.
$$

between the $K$-theory of the twisted group $C^{*}$-algebra $C^{*}(S(\Lambda, V), \tilde{\sigma})$ and the $K$-theory of the untwisted $C^{*}(S(\Lambda, V))$.

Proof. The argument is similar to that used in [14, Corollary 2.2; 19,20]. The cocycle $\tilde{\sigma}$ is real in the sense of Definition 1.12 of [14], being of the form (4.8), with $\sigma$ of the exponential form $\sigma\left((n, m),\left(n^{\prime}, m^{\prime}\right)\right)=\exp \left(-\pi i \theta\left(m n^{\prime}-n m^{\prime}\right)\right)$. Thus, as in Corollary 1.13 of [14], the identification (5.3) follows using a homotopy $\exp \left(-t \pi i \theta\left(m n^{\prime}-n m^{\prime}\right)\right)$, with $t \in[0,1]$.

Notice in fact that for groups of the form $\Gamma=\mathbb{Z}^{2} \rtimes_{\varphi} \mathbb{Z}$, with $\varphi \in \mathrm{SL}_{2}(\mathbb{Z})$, all cocycles $\sigma: \Gamma \times \Gamma \rightarrow U(1)$ are real in the above sense. This was observed already in [25].

Lemma 5.3. Let $\sigma \in Z^{2}(\Gamma, U(1))$ be a cocycle. Then $\sigma$ is cohomologous to a real cocycle, that is, to an element of $Z^{2}(\Gamma, U(1))$ that is of the form $\exp (2 \pi i \zeta)$ for $\zeta \in Z^{2}(\Gamma, \mathbb{R})$.

Proof. We can see it easily as in Section 2.2 of [19], by considering the exact sequence of coefficient groups

$$
1 \longrightarrow \mathbb{Z} \stackrel{\iota}{\rightarrow} \mathbb{R} \stackrel{\exp (2 \pi i \cdot)}{\longrightarrow} U(1) \longrightarrow 1
$$


and the long exact cohomology sequence

$$
\begin{aligned}
& \cdots \longrightarrow H^{2}(\Gamma, \mathbb{Z}) \longrightarrow H^{2}(\Gamma, \mathbb{R}) \stackrel{\exp (2 \pi i \cdot)_{*}}{\longrightarrow} H^{2}(\Gamma, U(1)) \\
& \stackrel{\delta}{\longrightarrow} H^{3}(\Gamma, \mathbb{Z}) \stackrel{\iota_{*}}{\longrightarrow} H^{3}(\Gamma, \mathbb{R}) \longrightarrow \cdots
\end{aligned}
$$

Since in our case, for $\Gamma=S(\Lambda, V)$, we have $E \Gamma=\mathbb{R}^{2} \rtimes \mathbb{R}$ and $B \Gamma=X_{\epsilon}$, we see that

$$
H^{2}(\Gamma, \mathbb{Z})=H^{2}\left(X_{\epsilon}, \mathbb{Z}\right)=H_{1}\left(X_{\epsilon}, \mathbb{Z}\right)=\mathbb{Z} \oplus \Lambda /(1-A) \Lambda
$$

and

$$
H^{3}(\Gamma, \mathbb{Z})=H^{3}\left(X_{\epsilon}\right)=\mathbb{Z}
$$

We then see that in the sequence (5.4) the map $\iota_{*}$ is injective so that $\delta=0$. Thus, all elements in $H^{2}(\Gamma, U(1))$ come from $H^{2}(\Gamma, \mathbb{R})$ via the exponential map.

In fact, we do not need this general fact, as the cocycle we are using is already constructed in the desired exponential form, but we stated it here for completeness.

\subsection{Thom isomorphism, homotopy quotients and Baum-Connes}

It is known that the group $S(\Lambda, V)$ satisfies the Baum-Connes conjecture (with coefficients). In fact, the group $\mathrm{SL}_{2}(\mathbb{Z})$ is known to satisfy the BaumConnes conjecture with coefficients, hence by [8] so does the group $\mathbb{Z}^{2} \rtimes_{\varphi_{\epsilon}} \mathbb{Z}$ with $\varphi_{\epsilon} \in \mathrm{SL}_{2}(\mathbb{Z})$.

This means that the Kasparov assembly map is an isomorphism, hence the $K$-theory of the $C^{*}$-algebra $C^{*}(S(\Lambda, V))$ can be computed in terms of the geometric $K$-theory of the homotopy quotient $\underline{B} \Gamma$, the classifying space for proper actions (cf. [4]). This relates directly the analytic $K$-theory of the $C^{*}$-algebra to the topological $K$-theory of the 3 -manifold $X_{\epsilon}$.

Lemma 5.4. The Kasparov assembly map for $C^{*}(S(\Lambda, V))$ gives an isomorphism

$$
\begin{aligned}
\mu: K^{1}\left(X_{\epsilon}\right) \stackrel{\cong}{\longrightarrow} K_{0}\left(C^{*}(S(\Lambda, V))\right), \\
\mu: K^{0}\left(X_{\epsilon}\right) \stackrel{\cong}{\longrightarrow} K_{1}\left(C^{*}(S(\Lambda, V))\right) .
\end{aligned}
$$


Proof. In our case, the space $\underline{E} \Gamma$ is the solvable Lie group $S\left(\mathbb{R}^{2}, \mathbb{R}\right)=\mathbb{R}^{2} \rtimes \mathbb{R}$ and the homotopy quotient $\underline{B} \Gamma=\Gamma \backslash \underline{E} \Gamma$ is the 3-manifold $X_{\epsilon}=S(\Lambda, V) \backslash$ $S\left(\mathbb{R}^{2}, \mathbb{R}\right)$. This can be identified with the mapping torus

$$
X_{\epsilon}=T^{2} \times[0,1] /((x, y), 0) \sim\left(A_{\epsilon}(x, y), 1\right)
$$

For a mapping torus, the Thom isomorphism [10] gives the identification

$$
K_{i+1}\left(C\left(X_{\epsilon}\right)\right)=K_{i}\left(C\left(T^{2}\right) \rtimes_{A_{\epsilon}} \mathbb{Z}\right) .
$$

Moreover, the $C^{*}$-algebra $C\left(T^{2}\right) \rtimes_{A_{\epsilon}} \mathbb{Z}$ is identified with $C^{*}(\Lambda) \rtimes V$ by the Fourier transform, which identifies $C\left(T^{2}\right)=C^{*}(\Lambda)$ for $T^{2}=\mathbb{R}^{2} / \Lambda$. The algebra $C^{*}(\Lambda) \rtimes V$ is then isomorphic to $C^{*}(S(\Lambda, V))$, by the same argument of Proposition 4.4 in the untwisted case.

\section{Twisted index theorem, $K$-theory and the range of the trace}

As we have seen, the 2-cocycle $\tilde{\sigma}$ on $\Gamma=S(\Lambda, V)$ is of the form $\tilde{\sigma}=\exp (2 \pi i \zeta)$, with $\zeta \in H^{2}(\Gamma, \mathbb{R})$. Upon identifying $H^{2}(\Gamma, \mathbb{R})=H^{2}(B \Gamma, \mathbb{R})=H^{2}\left(X_{\epsilon}, \mathbb{R}\right)$, we can identify the 2 -cocycle $\zeta$ with a closed 2 -form $\omega_{\epsilon}$ on the 3 -manifold $X_{\epsilon}$. We denote by $\tilde{\omega}_{\epsilon}$ its pullback to the universal covering $\tilde{X}_{\epsilon}=S\left(\mathbb{R}^{2}, \mathbb{R}\right)$. This is a $\Gamma$-invariant 2 -form, $\gamma^{*} \omega_{\epsilon}=\omega_{\epsilon}$, which we previously interpreted as a magnetic field.

Lemma 6.1. The real 2-cocycle $\zeta \in H^{2}(\Gamma, \mathbb{R})$ with $\tilde{\sigma}=\exp (2 \pi i \zeta)$ is given by

$$
\zeta((\lambda, k),(\eta, r))=\frac{1}{4 \pi i} \int_{\mathcal{R}} \omega
$$

where $\omega$ is the closed 2-form on $T^{2}=\mathbb{R}^{2} / \Lambda$ associated to the cocycle $\sigma$ on $\Lambda$, with magnetic flux $\int_{T^{2}} \omega=2 \pi i \theta\left(\theta^{\prime}-\theta\right)^{-1}$, and $\mathcal{R} \subset \mathbb{R}^{2}$ is the oriented parallelogram with vertices

$$
\left\{0, A_{\epsilon}^{k}(\eta), \lambda, \lambda+A_{\epsilon}^{k}(\eta)\right\}
$$

Proof. On $\tilde{X}_{\epsilon}$ the form $\tilde{\omega}_{\epsilon}$ is exact, hence we have a global magnetic potential $\chi_{\epsilon}$ with $\tilde{\omega}_{\epsilon}=d \chi_{\epsilon}$ and $d\left(\chi_{\epsilon}-\gamma^{*} \chi_{\epsilon}\right)=0$, or $\chi_{\epsilon}-\gamma^{*} \chi_{\epsilon}=d \phi_{\gamma}$, as before, where 
the $\phi_{\gamma}$ recovers the cocycle $\tilde{\sigma}$ by the formula

$$
\tilde{\sigma}\left(\gamma, \gamma^{\prime}\right)=\exp \left(-\phi_{\gamma}\left(\gamma^{\prime} x_{0}\right)\right)=\exp \left(\int_{x_{0}}^{\gamma^{\prime} x_{0}} \gamma^{*} \chi_{\epsilon}-\chi_{\epsilon}\right) \text {. }
$$

We know from Lemma 4.3 that the cocycle $\tilde{\sigma}$ has the form $\tilde{\sigma}_{u}((\lambda, k),(\eta, r))=$ $\sigma_{u}\left(\lambda, A_{\epsilon}^{k}(\eta)\right)$, for $u=\theta\left(\theta^{\prime}-\theta\right)^{-1}$, so that we have

$$
\zeta((\lambda, k),(\eta, r))=\frac{\theta\left(\theta^{\prime}-\theta\right)^{-1}}{2} A_{\epsilon}^{k}(\eta) \wedge \lambda,
$$

that is, $\zeta((\lambda, k),(\eta, r))=\xi\left(\lambda, A_{\epsilon}^{k}(\eta)\right)$, where

$$
\xi\left(\lambda, A_{\epsilon}^{k}(\eta)\right)=\frac{1}{2 \pi i} \int_{0}^{A_{\epsilon}^{k}(\eta)} U_{\lambda}^{*} \chi-\chi .
$$

Here $\chi$ is the magnetic potential on $\mathbb{R}^{2}$ associated to the closed 2 -form $\omega$ with

$$
\int_{T^{2}} \omega=2 \pi i \theta\left(\theta^{\prime}-\theta\right)^{-1}
$$

Let then $\mathcal{R}$ denote the oriented parallelogram in $\mathbb{R}^{2}$ with vertices as in (6.2). We have

$$
\frac{1}{2 \pi i} \int_{\mathcal{R}} \omega=\frac{1}{2 \pi i} \int_{0}^{A_{\epsilon}^{k}(\eta)}\left(U_{\lambda}^{*} \chi-\chi\right)-\frac{1}{2 \pi i} \int_{0}^{\lambda}\left(U_{A_{\epsilon}^{k}(\eta)}^{*} \chi-\chi\right) .
$$

Using the fact that $\xi(\eta, \lambda)=-\xi(\lambda, \eta)$, this gives

$$
\frac{1}{2 \pi i} \int_{\mathcal{R}} \omega=2 \zeta((\lambda, k),(\eta, r)) .
$$

\subsection{Spectral flow and odd Chern character}

An element of $K_{1}\left(C\left(X_{\epsilon}\right)\right)$ can be viewed as the class $[g]$ of $g \in U_{N}\left(C\left(X_{\epsilon}\right)\right)$, which we can see as a differentiable map $g: X_{\epsilon} \rightarrow \mathrm{GL}_{N}(\mathbb{C})$. We proceed as 
in [15] and we consider the associated 1-form

$$
\beta(g)=g^{-1} d g \in \Omega^{1}\left(X_{\epsilon}, g l_{N}(\mathbb{C})\right) .
$$

The corresponding family of connections $\nabla_{u}=d+u \beta(g)$ on the trivial bundle $X_{\epsilon} \times \mathbb{C}^{N}$ determines a closed Chern-Simons form

$$
\operatorname{Ch}(g):=c s(d, d+\beta(g))=\int_{0}^{1} \operatorname{Tr}\left(\frac{d}{d u}\left(\nabla_{u}\right) e^{\nabla_{u}^{2}}\right) d u,
$$

which gives the odd Chern character $\operatorname{Ch}(g)$. As shown in [15], this has an expression as an odd differential form

$$
\operatorname{Ch}(g)=\sum_{k=0}^{\infty}(-1)^{k} \frac{k !}{(2 k+1) !} \operatorname{Tr}\left(\beta(g)^{2 k+1}\right) .
$$

One then has, see [15], that the pairing

$$
\langle D,[g]\rangle=S F\left(D, g^{-1} D g\right)
$$

of an odd Fredholm module $(\mathcal{H}, D)$ with $[g] \in K_{1}$ is given by the spectral flow along $D_{u}=(1-u) D+u g^{-1} D g$. In the case where $D=\not \partial$ is the Dirac operator of a compact spin manifold, this is computed by the Atiyah-PatodiSinger index formula [3]. In our case, this gives

$$
S F\left(\not \partial, g^{-1} \not \partial g\right)=-\frac{1}{(2 \pi i)^{2}} \int_{X_{\epsilon}} \hat{A}\left(X_{\epsilon}\right) \mathrm{Ch}(g) .
$$

\subsection{Twisted index theorem}

We need the twisted version of (6.9) above. Let $\not \partial=\not_{X_{\epsilon}}$ be the Dirac operator on $X_{\epsilon}$, and let $\tilde{\not}$ be its lift to the universal cover $\tilde{X}_{\epsilon}=S\left(\mathbb{R}^{2}, \mathbb{R}\right)$. We then consider the twisting $\tilde{\phi} \otimes \nabla$ of the operator $\tilde{\not}$ by the hermitian connection $\nabla=d+i \eta_{\epsilon}$ on the trivial line bundle on $\tilde{X}_{\epsilon}$, with $\eta_{\epsilon}$ the 1-form giving the magnetic potential $d \eta_{\epsilon}=\omega_{\epsilon}$ on $\tilde{X}_{\epsilon}$.

While the operator $\tilde{\partial}$ is $\Gamma$-invariant, with $\Gamma=S(\Lambda, V)$, the twisted operator $\tilde{\partial} \otimes \nabla$ is only invariant under the projective action $(\Gamma, \tilde{\sigma})$ of the magnetic translations $R_{\gamma}^{\tilde{\sigma}}$.

Consider then the 1-parameter family of operators $D_{u}=\tilde{\phi}_{u} \otimes \nabla$, where $\tilde{\phi}_{u}=(1-u) \tilde{\phi}+u g^{-1} \tilde{\not} g$, for $[g] \in K^{1}\left(X_{\epsilon}\right)$ and the associated operator $\mathcal{D}_{g}=$ $\frac{\partial}{\partial u}+D_{u}$ on $\tilde{X}_{\epsilon} \times[0,1]$, which we can extend to $\tilde{X}_{\epsilon} \times \mathbb{R}($ cf. $[3$, p. 95]). 
Theorem 6.2. The range of the trace on $K_{0}\left(C^{*}(S(\Lambda, V), \tilde{\sigma})\right)$ is given by

$$
[\operatorname{tr}]\left(\mu_{\tilde{\sigma}}[g]\right)=\frac{-1}{(2 \pi i)^{2}} \int_{X_{\epsilon}} \hat{A} e^{\omega_{\epsilon}} \operatorname{Ch}(g)
$$

where $\mu_{\tilde{\sigma}}: K^{1}\left(X_{\epsilon}\right) \rightarrow K_{0}\left(C^{*}(S(\Lambda, V), \tilde{\sigma})\right)$ is the (twisted) Kasparov isomorphism, $[g] \in K^{1}\left(X_{\epsilon}\right)$ and $\omega_{\epsilon}$ is the closed 2-form on $X_{\epsilon}$ associated to the cocycle $\tilde{\sigma}$.

Proof. We let $P^{ \pm}$be the projections on the $L^{2}$-kernel of $\mathcal{D}_{g} \mathcal{D}_{g}^{*}$ and $\mathcal{D}_{g}^{*} \mathcal{D}_{g}$, respectively, namely

$$
\mathcal{D}_{g} P^{+}=0, \quad \mathcal{D}_{g}^{*} P^{-}=0 .
$$

The $P^{ \pm}$have smooth kernels $P^{ \pm}(x, y)$ and the $(\Gamma, \tilde{\sigma})$-invariance of $\tilde{\partial} \otimes \nabla$ implies that

$$
e^{-i \phi_{\gamma}(x)} P^{ \pm}(\gamma x, \gamma y) e^{i \phi_{\gamma}(y)}=P^{ \pm}(x, y)
$$

which implies that $P^{ \pm}(x, x)$ is $\Gamma$-invariant, for $\Gamma=S(\Lambda, V)$.

We proceed as in [1] and consider the von Neumann trace

$$
\operatorname{tr}\left(P^{ \pm}\right)=\int_{X_{\epsilon} \times S^{1}} \operatorname{tr} P^{ \pm}((x, t),(x, t)) d x d t
$$

where $\operatorname{tr} P^{ \pm}(x, x)$ is the pointwise trace. The $L^{2}$-index of $\mathcal{D}_{g}$ is given by

$$
\operatorname{Ind}_{L^{2}}\left(\mathcal{D}_{g}\right)=\operatorname{tr}\left(P^{+}\right)-\operatorname{tr}\left(P^{-}\right)
$$

We define $\bar{P}^{ \pm}$by the smooth kernels

$$
\bar{P}^{ \pm}(x, y)=\int_{S^{1}} \operatorname{tr} P^{ \pm}((x, t),(y, t)) d t
$$

These satisfy $\operatorname{tr}\left(\bar{P}^{ \pm}\right)=\operatorname{tr}\left(P^{ \pm}\right)$by

$$
\int_{X_{\epsilon} \times S^{1}} \operatorname{tr} P^{ \pm}((x, t),(x, t)) d x d t=\int_{X_{\epsilon}} \operatorname{tr} \bar{P}^{ \pm}(x, x) d x .
$$


The projections $\bar{P}^{ \pm}$are in the von Neumann algebra $\mathcal{U}(\Gamma, \tilde{\sigma})$. After adding a compact perturbation in $C_{r}^{*}(\Gamma, \tilde{\sigma})$ one obtains a well-defined index (cf. $[23,28]$ ),

$$
\operatorname{Ind}_{(\Gamma, \tilde{\sigma})}\left(\mathcal{D}_{g}\right)=\left[\bar{P}^{+}\right]-\left[\bar{P}^{-}\right] \in K_{0}\left(C_{r}^{*}(\Gamma, \tilde{\sigma})\right) .
$$

The (twisted) Kasparov map $\mu: K^{1}\left(X_{\epsilon}\right) \rightarrow K_{0}\left(C^{*}(\Gamma, \tilde{\sigma})\right)$ is given by

$$
\mu_{\tilde{\sigma}}[g]=\operatorname{Ind}_{(\Gamma, \tilde{\sigma})}\left(\mathcal{D}_{g}\right)
$$

We obtain in this way that

$$
\operatorname{Ind}_{L^{2}}\left(\mathcal{D}_{g}\right)=\operatorname{tr}\left(\bar{P}^{+}\right)-\operatorname{tr}\left(\bar{P}^{-}\right)=\operatorname{tr}\left(\operatorname{Ind}_{(\Gamma, \tilde{\sigma})}\left(\mathcal{D}_{g}\right)\right)
$$

Consider the heat kernel $e^{-t \mathcal{D}^{2}}$, where

$$
\mathcal{D}=\left(\begin{array}{cc}
0 & \mathcal{D}_{g}^{*} \\
\mathcal{D}_{g} & 0
\end{array}\right) \quad \text { with } \mathcal{D}^{2}=\left(\begin{array}{cc}
\mathcal{D}_{g}^{*} \mathcal{D}_{g} & 0 \\
0 & \mathcal{D}_{g} \mathcal{D}_{g}^{*}
\end{array}\right)
$$

We have

$$
\lim _{t \rightarrow \infty} \operatorname{tr}_{s}\left(e^{-t \mathcal{D}^{2}}\right)=\operatorname{tr}\left(P^{+}\right)-\operatorname{tr}\left(P^{-}\right)
$$

and

$$
\frac{\partial}{\partial t} \operatorname{tr}_{s}\left(e^{-t \mathcal{D}^{2}}\right)=-\operatorname{tr}_{s}\left(\mathcal{D}^{2} e^{-t \mathcal{D}^{2}}\right)=\operatorname{tr}_{s}\left(\left[\mathcal{D} e^{-t \mathcal{D}^{2}}, \mathcal{D}\right]\right)=0
$$

Thus

$$
\begin{aligned}
& \operatorname{tr}\left(P^{+}\right)-\operatorname{tr}\left(P^{-}\right)=\lim _{t \rightarrow \infty} \operatorname{tr}_{s}\left(e^{-t \mathcal{D}^{2}}\right)=\lim _{t \rightarrow 0} \operatorname{tr}_{s}\left(e^{-t \mathcal{D}^{2}}\right) \\
& =\frac{-1}{(2 \pi i)^{2}} \int_{X_{\epsilon} \times S^{1}} \hat{A} \operatorname{Ch}\left(\nabla_{u}\right)=\frac{-1}{(2 \pi i)^{2}} \int_{X_{\epsilon}} \hat{A} e^{\omega_{\epsilon}} \operatorname{Ch}(g),
\end{aligned}
$$

where $\operatorname{Ch}\left(\nabla_{u}\right)=\operatorname{tr}\left(\beta e^{(d+u \beta)^{2}}\right)$ for $\beta=g^{-1} d g$, with $\int_{S^{1}} \operatorname{Ch}\left(\nabla_{u}\right)=\operatorname{Ch}(g)$.

\subsection{Range of the trace}

Using the twisted index theorem we can then compute explicitly the range of the trace on $K_{0}\left(C^{*}(S(\Lambda, V), \tilde{\sigma})\right)$. We obtain the following result.

Proposition 6.3. The range of the trace on $K_{0}\left(C^{*}(S(\Lambda, V), \tilde{\sigma})\right)$ is

$$
[\operatorname{tr}]\left(K_{0}\left(C^{*}(S(\Lambda, V), \tilde{\sigma})\right)\right)=\mathbb{Z}+\mathbb{Z} \theta\left(\theta^{\prime}-\theta\right)^{-1} .
$$


Proof. Since $X_{\epsilon}$ is a 3-manifold, when we expand the terms in the cohomological formula (6.10) as

$$
\begin{gathered}
\hat{A}\left(X_{\epsilon}\right)=1-\frac{1}{24} p_{1}\left(X_{\epsilon}\right)+\cdots, \\
e^{\omega_{\epsilon}}=1+\omega_{\epsilon}+\frac{1}{2} \omega_{\epsilon}^{2}+\cdots, \\
\mathrm{Ch}(g)=-\frac{1}{6} \operatorname{Tr}(\beta(g))+\frac{1}{5 !} \operatorname{Tr}\left(\beta^{3}(g)\right)+\cdots,
\end{gathered}
$$

only the terms of the wedge product $\hat{A}\left(X_{\epsilon}\right) e^{\omega} \mathrm{Ch}(g)$ that give differential forms of order up to 3 can contribute nontrivially.

Thus, we obtain the terms

$$
\frac{1}{(2 \pi)^{2}} \int_{X_{\epsilon}}\left(\frac{-1}{6} \operatorname{Tr}(\beta(g)) \wedge \omega+\frac{1}{5 !} \operatorname{Tr}\left(\beta(g)^{3}\right)\right) .
$$

The term

$$
\frac{1}{(2 \pi)^{2}} \int_{X_{\epsilon}} \frac{1}{5 !} \operatorname{Tr}\left(\beta(g)^{3}\right)=\frac{1}{(2 \pi)^{2}} \int_{X_{\epsilon}} \operatorname{Ch}(g)
$$

is the term one would find in the untwisted case, and it gives the untwisted odd Chern character.

For the remaining term

$$
\frac{1}{(2 \pi)^{2}} \frac{-1}{6} \int_{X_{\epsilon}} \operatorname{Tr}(\beta(g)) \wedge \omega_{\epsilon}
$$

the range as $[g]$ varies in $K_{1}\left(C\left(X_{\epsilon}\right)\right)$ is given by $\mathbb{Z} R(\omega)$, where $R(\omega)$ is the range of the linear form

$$
T_{\omega}:[g] \longmapsto \frac{1}{(2 \pi)^{2}} \frac{-1}{6} \int_{X_{\epsilon}} \operatorname{Tr}(\beta(g)) \wedge \omega_{\epsilon} \in \mathbb{R} .
$$

First notice that, with the notation $\mathrm{Ch}_{1}(g)=\frac{-1}{6} \operatorname{Tr}(\beta(g))$, we have

$$
\int_{C} \operatorname{Ch}_{1}(g)=2 \pi i \operatorname{deg}\left(\left.g\right|_{C}\right) \in 2 \pi i \mathbb{Z},
$$

for $C \in H_{1}\left(X_{\epsilon}, \mathbb{Z}\right)$. Thus, we obtain

$$
\frac{1}{2 \pi i} \int_{X_{\epsilon}} \mathrm{Ch}_{1}(g) \wedge P D(C) \in \mathbb{Z}
$$


for $P D(C) \in H^{2}\left(X_{\epsilon}, \mathbb{Z}\right) \hookrightarrow H^{2}\left(X_{\epsilon}, \mathbb{R}\right)$. Now consider the explicit description of the 2 -form $\omega_{\epsilon}$ given in Lemma 6.1 above. We can write

$$
\omega_{\epsilon}=2 \pi i \theta\left(\theta^{\prime}-\theta\right)^{-1} \bar{\omega}_{\epsilon}
$$

where $\bar{\omega}_{\epsilon} \in H^{2}\left(X_{\epsilon}, \mathbb{Z}\right)$ is given by

$$
\bar{\omega}_{\epsilon}(v, w)=A_{\epsilon}^{k}(\eta) \wedge \lambda
$$

for $v=((0,0),(\lambda, k))$ and $w=((0,0),(\eta, r))$. Thus, we see that we can write

$$
\begin{aligned}
\frac{1}{(2 \pi)^{2}} \int_{X_{\epsilon}} \mathrm{Ch}_{1}(g) \wedge \omega_{\epsilon} & =-\frac{1}{2 \pi i} \theta\left(\theta^{\prime}-\theta\right)^{-1} \int_{X_{\epsilon}} \mathrm{Ch}_{1}(g) \wedge \bar{\omega}_{\epsilon} \\
& =-\frac{1}{2 \pi i} \theta\left(\theta^{\prime}-\theta\right)^{-1} \int_{P D\left(\bar{\omega}_{\epsilon}\right)} \mathrm{Ch}_{1}(g) \\
& =\theta\left(\theta^{\prime}-\theta\right)^{-1} \operatorname{deg}\left(\left.g\right|_{P D\left(\bar{\omega}_{\epsilon}\right)}\right) \in \theta\left(\theta^{\prime}-\theta\right)^{-1} \mathbb{Z}
\end{aligned}
$$

\section{Isospectral deformations and spectral triples}

In noncommutative geometry, the analog of Riemannian structures is provided by the formalism of spectral triples [11]. A spectral triple on a noncommutative space $\mathcal{A}$ (where $\mathcal{A}$ is a $C^{*}$-algebra) consists of the data $\left(\mathcal{A}_{\infty}, \mathcal{H}, D\right)$ of a dense involutive subalgebra $\mathcal{A}_{\infty}$, a representation $\pi: \mathcal{A} \rightarrow \mathcal{B}(\mathcal{H})$ as bounded operators on a Hilbert space $\mathcal{H}$ and a self-adjoint operator $D$ on $\mathcal{H}$, with compact resolvent, satisfying the compatibility condition

$$
[D, \pi(a)] \in \mathcal{B}(\mathcal{H}), \quad \forall a \in \mathcal{A}_{\infty}
$$

In particular, in the commutative case, to a Riemannian spin-manifold $X$ one can associate a canonical spectral triple $\left(C^{\infty}(X), L^{2}(X, S), \not \partial\right)$. A reconstruction theorem $[12,26]$ shows that a spectral triple where the algebra is abelian, which satisfies a list of axioms, is the canonical spectral triple of a Riemannian spin-manifold.

In our case, we have a spectral triple associated to the 3 -manifold $X_{\epsilon}$, where the spinor bundle is a complex 2-plane bundle and the Dirac operator 
can be written in the form

$$
\partial_{X_{\epsilon}}=c(d t) \frac{\partial}{\partial t}+c\left(e^{t} d x\right) \frac{\partial}{\partial x}+c\left(e^{-t} d y\right) \frac{\partial}{\partial y},
$$

where $\left\{d t, e^{t} d x, e^{-t} d y\right\}$ is the basis of the cotangent bundle of $S\left(\mathbb{R}^{2}, \mathbb{R}, \epsilon\right)=$ $\mathbb{R}^{2} \rtimes_{A_{\epsilon}} \mathbb{R}$ and $c(\omega)$ denotes the Clifford multiplication by the 1-form $\omega$.

More explicitly, (7.2) is of the form

$$
\not_{X_{\epsilon}}=\frac{\partial}{\partial t} \sigma_{0}+e^{t} \frac{\partial}{\partial x} \sigma_{1}+e^{-t} \frac{\partial}{\partial y} \sigma_{2}=\left(\begin{array}{cc}
\frac{\partial}{\partial t} & e^{-t} \frac{\partial}{\partial y}-i e^{t} \frac{\partial}{\partial x} \\
e^{-t} \frac{\partial}{\partial y}+i e^{t} \frac{\partial}{\partial x} & -\frac{\partial}{\partial t}
\end{array}\right),
$$

where $\sigma_{i}$, for $i=0,1,2$, are the Pauli matrices.

Our purpose here is to show that this commutative spectral triple can be deformed isospectrally to a spectral triple for the noncommutative tori $\mathbb{T}_{\Lambda, i}$.

\subsection{The Connes-Landi isospectral deformations}

We consider the problem from the point of view of the Connes-Landi isospectral deformations [13]. This provides a general procedure to deform commutative spectral triples to noncommutative ones isospectrally, for manifolds with isometric torus actions.

We recall briefly the construction of isospectral deformations, in a version that is best adapted to our setting.

Suppose given a spectral triple $\left(C^{\infty}(X), L^{2}(X, S), \not{ }_{X}\right)$ associated to a compact Riemannian spin-manifold $X$. Assume that the manifold $X$ has an action of a torus $T^{2}$ by isometries, $T^{2} \subset \operatorname{Isom}(X)$. Then one considers a noncommutative algebra $\mathcal{A}_{\theta}$, depending on a real parameter $\theta \in \mathbb{R}$, which is obtained by decomposing the operators $\pi(f) \in \mathcal{B}(\mathcal{H})$, for $f \in C^{\infty}(X)$ and $\mathcal{H}=L^{2}(X, S)$ according to their weighted components

$$
\pi(f)=\sum_{n, m \in \mathbb{Z}} \pi\left(f_{n, m}\right),
$$

where

$$
\alpha_{\tau}\left(\pi\left(f_{n, m}\right)\right)=e^{2 \pi i\left(n \tau_{1}+m \tau_{2}\right)} \pi\left(f_{n, m}\right), \quad \forall \tau=\left(\tau_{1}, \tau_{2}\right) \in T^{2},
$$

for

$$
\alpha_{\tau}(T)=U(\tau) T U(\tau)^{*}, \quad \forall T \in \mathcal{B}(\mathcal{H}), \quad \forall \tau \in T^{2},
$$


with $U(\tau)$ the unitary transformations implementing the $T^{2}$-action on $\mathcal{H}=L^{2}(X, S)$ by

$$
U(\tau) \psi(x)=\psi\left(\tau^{-1}(x)\right)
$$

Let $L_{1}$ and $L_{2}$ denote the infinitesimal generators of the action

$$
U(\tau)=\exp (2 \pi i \tau L)=\exp \left(2 \pi i\left(\tau_{1} L_{1}+\tau_{2} L_{2}\right)\right)
$$

We consider then the subalgebra of $\mathcal{B}(\mathcal{H})$ generated by the operators of the form

$$
\pi_{\xi_{1}, \xi_{2}}(f)=\sum_{n, m} \pi\left(f_{n, m}\right) e^{-2 \pi i\left(\xi_{1} n L_{2}+\xi_{2} m L_{1}\right)}
$$

where $\xi_{1}$ and $\xi_{2}$ are two real parameters.

Lemma 7.1. For homogeneous operators $\pi(f)_{n, m}$ define the deformed product

$$
f_{n, m} * \xi_{1}, \xi_{2} h_{k, r}:=e^{-2 \pi i\left(\xi_{1} n r+\xi_{2} m k\right)} f_{n, m} h_{k, r}
$$

The product of operators of the form (7.8) satisfies

$$
\pi_{\xi_{1}, \xi_{2}}\left(f_{n, m}\right) \pi_{\xi_{1}, \xi_{2}}\left(h_{k, r}\right)=\pi_{\xi_{1}, \xi_{2}}\left(f_{n, m} *_{\xi_{1}, \xi_{2}} h_{k, r}\right)
$$

Proof. One checks directly that the operator product $\pi\left(f_{\xi_{1}, \xi_{2}}\right) \pi\left(h_{\xi_{1}, \xi_{2}}\right)$ is given in components by

$$
\pi\left(f_{n, m}\right) *_{\xi_{1}, \xi_{2}} \pi\left(h_{k, r}\right)=e^{-2 \pi i\left(\xi_{1} n r+\xi_{2} m k\right)} \pi\left(f_{n, m}\right) \pi\left(h_{k, r}\right)
$$

One can recognize in (7.9) the convolution product of the twisted group $C^{*}$-algebra $C^{*}\left(\mathbb{Z}^{2}, \sigma\right)$ with the cocycle

$$
\sigma((n, m),(k, r))=\exp \left(-2 \pi i\left(\xi_{1} n r+\xi_{2} m k\right)\right)
$$

As shown in [13], the operators (7.8) have bounded commutators with the Dirac operator. In fact, since $T^{2}$ acts by isometries, the Dirac operator 
satisfies

$$
U(\tau) D U(\tau)^{*}=D
$$

i.e., it is of bidegree $(0,0)$. Thus, one sees that the commutators give

$$
\begin{aligned}
{\left[D, \pi_{\xi_{1}, \xi_{2}}(f)\right] } & =\sum_{n, m}\left[D, \pi(f)_{n, m} e^{-2 \pi i\left(\xi_{1} n L_{2}+\xi_{2} m L_{1}\right)}\right] \\
& =\sum_{n, m}[D, \pi(f)]_{n, m} e^{-2 \pi i\left(\xi_{1} n L_{2}+\xi_{2} m L_{1}\right)}
\end{aligned}
$$

which is still a bounded operator on $\mathcal{H}$.

We consider in particular the case where $\xi_{2}=u / 2=-\xi_{1}$. We denote by $\mathcal{A}_{u}=C^{\infty}(X)_{u}$ the deformed algebra, that is, the algebra generated by the (7.8). The deformed spectral triple is given by the data $\left(\mathcal{A}_{u}, L^{2}(X, S), \not_{X}\right)$.

\subsection{Noncommutative solvmanifolds}

We apply the procedure described above to obtain an isospectral deformation of the solvmanifold $X_{\epsilon}$, which corresponds to deforming the fiber tori to noncommutative tori.

The canonical spectral triple for $X_{\epsilon}$ consists of the data $\left(C^{\infty}\left(X_{\epsilon}\right)\right.$, $\left.L^{2}\left(X_{\epsilon}, S\right), \partial_{X_{\epsilon}}\right)$, with the Dirac operator of the form (7.2).

There is a torus action on $X_{\epsilon}$ by isometries, which consists of translations along the fibers of the fibration $T^{2} \rightarrow X_{\epsilon} \rightarrow S^{1}$. This acts on spinors by unitaries

$$
U(\tau) \psi((x, y), t)=\psi\left(\left(x+e^{t} \tau_{1}, y+e^{-t} \tau_{2}\right), t\right)
$$

for $\tau \in T^{2}=\mathbb{R}^{2} / \Lambda$ and $(x, y) \in T_{t}^{2}=\mathbb{R}^{2} / \Lambda_{t}$, the fiber over $t \in S^{1}$, with $\left(e^{t} \lambda_{1}, e^{-t} \lambda_{2}\right) \in \Lambda_{t}$, for $\left(\lambda_{1}, \lambda_{2}\right) \in \Lambda$.

The action clearly preserves the metric $d t^{2}+e^{t} d x^{2}+e^{-t} d y^{2}$, hence the Dirac operator (7.3) satisfies

$$
U(\tau) \partial_{X_{\epsilon}} U(\tau)^{*}=\partial_{X_{\epsilon}}
$$

The infinitesimal generators of the action $\alpha_{\tau}$ are the operators $2 \pi L_{1}=e^{t} \frac{\partial}{\partial x}$, $2 \pi L_{2}=e^{-t} \frac{\partial}{\partial y}$ with $U(\tau)=\exp \left(2 \pi i\left(\tau_{1} L_{1}+\tau_{2} L_{2}\right)\right)$.

We introduce the following notation. We denote by $E_{\lambda}$, for $\lambda \in \Lambda$, the function

$$
E_{\lambda}((x, y), t):=e^{2 \pi i\left\langle\Theta_{-t}(x, y), \lambda\right\rangle}
$$


where, as above, $\Theta_{-t}(x, y)=\left(e^{-t} x, e^{t} y\right)$ and $\langle(a, b), \lambda\rangle=a \lambda_{1}+b \lambda_{2}$. We also denote by $\Xi_{u}\left(\lambda, L_{1}, L_{2}\right)$ the operator

$$
\Xi_{u}\left(\lambda, L_{1}, L_{2}\right):=\exp \left(i \pi \frac{u}{\left(\theta^{\prime}-\theta\right)} \lambda \wedge\left(L_{1}, L_{2}\right)\right),
$$

acting on $\mathcal{H}=L^{2}\left(X_{\epsilon}, S\right)$.

Proposition 7.2. The deformed algebra $C^{\infty}\left(X_{\epsilon}\right)_{u}$, for $u \in \mathbb{R}$, is the $C^{*}$ subalgebra of $\mathcal{B}(\mathcal{H})$, with $\mathcal{H}=L^{2}\left(X_{\epsilon}, S\right)$ generated by the operators of the form

$$
\pi_{u}(f)=E_{\lambda} \Xi_{u}\left(\lambda, L_{1}, L_{2}\right)
$$

Proof. The induced action $\alpha: T^{2} \rightarrow \operatorname{Aut}\left(C^{\infty}\left(X_{\epsilon}\right)\right)$ defined by

$$
\pi\left(\alpha_{\tau}(f)\right)=U(\tau) \pi(f) U(\tau)^{*}
$$

is of the form $\alpha_{\tau}(f)((x, y), t)=f\left(\left(x+e^{t} \tau_{1}, y+e^{-t} \tau_{2}\right), t\right)$.

Thus, a homogeneous operator of bidegree $\lambda=\left(\lambda_{1}, \lambda_{2}\right)$ is in this case a function $f_{\lambda}((x, y), t)$ with the property that

$$
\alpha_{\tau}\left(f_{\lambda}\right)((x, y), t)=e^{2 \pi i\left(\lambda_{1} \tau_{1}+\lambda_{2} \tau_{2}\right)} f_{\lambda}((x, y), t)
$$

This condition is satisfied by functions of the form

$$
f_{\lambda}((x, y), t)=\exp \left(2 \pi i\left\langle\Theta_{-t}(x, y), \lambda\right\rangle\right)=\exp \left(2 \pi i\left(e^{-t} \lambda_{1} x+e^{t} \lambda_{2} y\right)\right)
$$

Under the change of variables

$$
\mathbb{Z}^{2} \longrightarrow \Lambda, \quad(n, m) \longmapsto \lambda=\left(n+m \theta, n+m \theta^{\prime}\right),
$$

the condition (7.14) corresponds to elements $f_{n, m}$ of bidegree $(n, m)$ for the corresponding action of $T^{2}=\mathbb{R}^{2} / \mathbb{Z}^{2}$. Thus, using this change of coordinates to pass in (7.8) from $\mathbb{Z}^{2}$ to $\Lambda$, we can see that elements of the deformed algebra of the form (7.8) correspond to elements of the form

$$
\sum_{\lambda} a_{\lambda} E_{\lambda} \Xi_{u}\left(\lambda, L_{1}, L_{2}\right)
$$

for $\xi_{2}=u / 2=-\xi_{1}$. 
Set $2 u_{\theta}=u /\left(\theta^{\prime}-\theta\right)$. The operators (7.13) act on spinors by

$$
\begin{aligned}
\left(\pi_{u}(f) \psi\right)((x, y), t) & =E_{\lambda}((x, y), t)\left(U\left(\left(-\lambda_{2} u_{\theta}, \lambda_{1} u_{\theta}\right)\right) \psi\right)((x, y), t) \\
& =e^{2 \pi i\left\langle\Theta_{-t}(x, y), \lambda\right\rangle} \psi\left(\left(x-e^{t} \lambda_{2} u_{\theta}, y+e^{-t} \lambda_{1} u_{\theta}\right), t\right) .
\end{aligned}
$$

Proposition 7.3. The operators

$$
\pi\left(R_{\lambda}^{\sigma}\right):=E_{\lambda} \Xi_{u}\left(\lambda, L_{1}, L_{2}\right)
$$

define a representation on $\mathcal{H}=L^{2}\left(X_{\epsilon}, S\right)$ of the noncommutative torus $C^{*}(\Lambda, \sigma)$, with the cocycle

$$
\sigma(\lambda, \eta)=\exp \left(2 \pi i u_{\theta} \lambda \wedge \eta\right)
$$

Proof. Notice that we have

$$
U\left(-\lambda_{2} u_{\theta}, \lambda_{1} u_{\theta}\right) e^{2 \pi i\left\langle\Theta_{-t}(x, y), \eta\right\rangle}=e^{2 \pi i u_{\theta} \lambda \wedge \eta} e^{2 \pi i\left\langle\Theta_{-t}(x, y), \eta\right\rangle} .
$$

Thus, we obtain

$$
\begin{aligned}
& e^{2 \pi i\left\langle\Theta_{-t}(x, y), \lambda\right\rangle} \Xi_{u}\left(\lambda, L_{1}, L_{2}\right) e^{2 \pi i\left\langle\Theta_{-t}(x, y), \eta\right\rangle} \Xi_{u}\left(\eta, L_{1}, L_{2}\right) \\
& =e^{2 \pi i u_{\theta} \lambda \wedge \eta} e^{2 \pi i\left\langle\Theta_{-t}(x, y), \lambda\right\rangle} e^{2 \pi i\left\langle\Theta_{-t}(x, y), \eta\right\rangle} \Xi_{u}\left(\lambda, L_{1}, L_{2}\right) \Xi_{u}\left(\eta, L_{1}, L_{2}\right) \\
& =e^{2 \pi i u_{\theta} \lambda \wedge \eta} e^{2 \pi i\left\langle\Theta_{-t}(x, y), \lambda+\eta\right\rangle} \Xi_{u}\left(\lambda+\eta, L_{1}, L_{2}\right) .
\end{aligned}
$$

This shows that the operators $\pi\left(R_{\lambda}^{\sigma}\right)$ satisfy the product rule

$$
\pi\left(R_{\lambda}^{\sigma}\right) \pi\left(R_{\eta}^{\sigma}\right)=\sigma(\lambda, \eta) \pi\left(R_{\lambda+\eta}^{\sigma}\right)
$$

for $\sigma(\lambda, \eta)=\exp \left(2 \pi i u_{\theta} \lambda \wedge \eta\right)$, which is the product rule of the twisted group algebra $C^{*}(\Lambda, \sigma)$.

We obtain in this way an isospectral noncommutative geometry given by the finitely summable spectral triple

$$
\left(C^{\infty}\left(X_{\epsilon}\right)_{u}, L^{2}\left(X_{\epsilon}, S\right), \not_{X_{\epsilon}}\right)
$$

Corollary 7.4. In the case $u=\theta$ and $u=\theta^{\prime}$, the isospectral deformation (7.18) defines a finitely summable spectral triple for the noncommutative tori $\mathbb{T}_{\Lambda, i}$, with dense subalgebra $\mathbb{C}(\Lambda, \sigma)$.

Proof. This is a direct consequence of Proposition 7.3 and the identifications of Corollary 4.2 of the $\mathbb{T}_{\Lambda, i}$ with twisted group $C^{*}$-algebras $C^{*}(\Lambda, \sigma)$. 
The representation (7.17) of $C^{*}(\Lambda, \sigma)$ extends to an action of $C^{*}(S(\Lambda, V), \tilde{\sigma})$, as follows. Let $U(k \log \epsilon)$ denote the unitary operator

$$
(U(k \log \epsilon) \psi)((x, y), t)=\psi\left(A_{\epsilon}^{k}(x, y), t\right)=\psi((x, y), t-k \log \epsilon)
$$

Proposition 7.5. The operators

$$
\pi\left(R_{(\lambda, k)}^{\tilde{\sigma}}\right):=E_{\lambda} \Xi_{u}\left(\lambda, L_{1}, L_{2}\right) U(k \log \epsilon)
$$

define a representation on $\mathcal{H}=L^{2}\left(X_{\epsilon}, S\right)$ of the twisted group $C^{*}$-algebra $C^{*}\left(\Lambda \rtimes_{\epsilon} V, \tilde{\sigma}\right)$, for the cocycle

$$
\tilde{\sigma}((\lambda, k),(\eta, r))=\exp \left(2 \pi i \frac{u_{\theta}}{2} \lambda \wedge A_{\epsilon}^{k}(\eta)\right)
$$

Proof. We have the identities

$$
\begin{aligned}
& \left(U(k \log \epsilon) E_{\eta}\right)=E_{A_{\epsilon}^{k}(\eta)}, \\
& \Xi_{u}\left(\lambda, L_{1}, L_{2}\right) E_{A_{\epsilon}^{k}(\eta)}=e^{2 \pi i u \lambda \wedge A_{\epsilon}^{k}(\eta)} E_{A_{\epsilon}^{k}(\eta)}, \\
& E_{\lambda} E_{A_{\epsilon}^{k}(\eta)}=E_{\lambda+A_{\epsilon}^{k}(\eta)}, \\
& U(k \log \epsilon) \Xi_{u}\left(\eta, L_{1}, L_{2}\right) U(r \log \epsilon)=\Xi_{u}\left(A_{\epsilon}^{k}(\eta), L_{1}, L_{2}\right) U((k+r) \log \epsilon) .
\end{aligned}
$$

These combine to give the composition rule

$$
\begin{aligned}
& E_{\lambda} \Xi_{u}\left(\lambda, L_{1}, L_{2}\right) U(k \log \epsilon) E_{\eta} \Xi_{u}\left(\eta, L_{1}, L_{2}\right) U(r \log \epsilon) \\
& \quad=\tilde{\sigma}((\eta, r),(\lambda, k)) E_{\lambda+A_{\epsilon}^{k}(\eta)} \Xi_{u}\left(\lambda+A_{\epsilon}^{k}(\eta), L_{1}, L_{2}\right) U((k+r) \log \epsilon) .
\end{aligned}
$$

\subsection{Unitary equivalences}

We begin by reformulating the data described above in an equivalent form by expanding in Fourier modes along the fiber tori as in [2].

Recall that the fiber over $t \in[0, \log \epsilon)$ is given by the torus $T_{t}^{2}=\mathbb{R}^{2} / \Lambda_{t}$, with $\Lambda_{t}=\Theta_{t}(\Lambda)$. Thus, if we denote by $(x, y)$, as above, the coordinates in $T_{t}^{2}$, we can write these as $(x, y)=\Theta_{t}(a, b)$, with $(a, b) \in T^{2}=\mathbb{R}^{2} / \Lambda$, the reference torus. 
This means writing the spinors $\psi((x, y), t)$ in the form

$$
\sum_{\lambda} \psi_{\lambda} e^{2 \pi i\langle(a, b), \lambda\rangle}=\sum_{\lambda} \psi_{\lambda} e^{2 \pi i\left\langle\Theta_{-t}(x, y), \lambda\right\rangle}=\sum_{\lambda} \psi_{\lambda} E_{\lambda} .
$$

The Dirac operator acts on $E_{\lambda}$ as

$$
\not_{X_{\epsilon}} E_{\lambda}=\left(\frac{\partial}{\partial t} \sigma_{0}+2 \pi i \lambda_{1} \sigma_{1}+2 \pi i \lambda_{2}\right) E_{\lambda} .
$$

The operators $\pi\left(R_{\eta}^{\sigma}\right)$ act as

$$
E_{\eta} \Xi_{u}\left(\eta, L_{1}, L_{2}\right) E_{\lambda}=e^{2 \pi i u \eta \wedge \lambda} E_{\eta+\lambda} .
$$

The commutators are bounded operators of the form

$$
\left[\not_{X_{\epsilon}}, \pi\left(R_{\eta}^{\sigma}\right)\right]=\left(\eta_{1} \sigma_{1}+\eta_{2} \sigma_{2}\right) R_{\eta}^{\sigma} .
$$

Thus, passing to Fourier modes in the fiber directions gives a unitarily equivalent spectral triple for the noncommutative tori $\mathbb{T}_{\Lambda, i}$, with

$$
\begin{aligned}
\hat{\partial}_{X_{\epsilon}} \psi_{\lambda} & =\left(\frac{\partial}{\partial t} \sigma_{0}+2 \pi i \lambda_{1} \sigma_{1}+2 \pi i \lambda_{2} \sigma_{2}\right) \psi_{\lambda} \\
\hat{\pi}\left(R_{\eta}^{\sigma}\right) \psi_{\lambda} & =\sigma(\eta, \lambda) \psi_{\lambda+\eta} .
\end{aligned}
$$

We then consider a second unitary equivalence, which, as in [2] adjusts for the possible signs of $\lambda_{1}$ and $\lambda_{2}$. Namely, we define the following unitary operator on the Hilbert space of the spinors $\psi_{\lambda}$. We set

$$
\mathcal{U} \psi_{\lambda}=\sigma_{\lambda} \psi_{\lambda}
$$

where $\sigma_{\lambda}$ is a product of Pauli matrices, where $\sigma_{i}$, for $i=1,2$, appears in the product if and only if $\lambda_{i}<0$. Then the Dirac operator transforms to the unitarily equivalent operator

$$
\mathcal{U} \hat{\partial}_{X_{\epsilon}} \mathcal{U}^{*}=\operatorname{sign}(N(\lambda))\left(\frac{\partial}{\partial t} \sigma_{0}+2 \pi i\left|\lambda_{1}\right| \sigma_{1}+2 \pi i\left|\lambda_{2}\right| \sigma_{2}\right) .
$$

The action of the $R_{\eta}^{\sigma}$ transform correspondingly to the operators

$$
\mathcal{U} \hat{\pi}\left(R_{\eta}^{\sigma}\right) \mathcal{U}^{*}: \sigma_{\lambda} \psi_{\lambda} \longmapsto \sigma_{\lambda+\eta} \psi_{\lambda+\eta} .
$$

We then perform the other unitary transformation used in [2]. To this purpose, let us fix a choice of a fundamental domain $\mathcal{F}_{V}$ for the action of 
$V$ on the lattice $\Lambda$. By this choice of a fundamental domain, we can write uniquely an element $\lambda \in \Lambda$ in the form $\lambda=A_{\epsilon}^{k}(\mu)$, for a $\mu \in \mathcal{F}_{V}$ and a $k \in \mathbb{Z}$.

For $\lambda=A_{\epsilon}^{k}(\mu) \neq 0$, consider then the time shift

$$
\tilde{\mathcal{U}}\left(\sigma_{\lambda} \psi_{\lambda}\right)(t)=\sigma_{\lambda} \psi_{\lambda}\left(t-\log \frac{\left|\mu_{1}\right|}{|N(\mu)|^{1 / 2}}\right),
$$

so that we have

$$
\tilde{\psi}_{\lambda}:=\tilde{\mathcal{U}}\left(\sigma_{\lambda} \psi_{\lambda}\right)=\sigma_{\lambda} \psi_{|N(\lambda)|^{1 / 2}\left(\operatorname{sign}\left(\lambda_{1}\right) \epsilon^{k}, \operatorname{sign}\left(\lambda_{2}\right) \epsilon^{-k}\right)}
$$

One obtains in this way a unitarily equivalent spectral triple for $\mathbb{T}_{\Lambda, i}$, with the Dirac operator

$$
\tilde{\partial}=\tilde{\not}^{(0)}+\sum_{\mu \in(\Lambda \backslash\{0\}) / V} \tilde{\partial}^{(\mu)}
$$

where

$$
\begin{aligned}
\tilde{\partial}^{(\mu)} \tilde{\psi}_{A_{\epsilon}^{k}(\mu)}= & \operatorname{sign}(N(\mu))|N(\mu)|^{1 / 2} \\
& \times\left(|N(\mu)|^{-1 / 2} \frac{\partial}{\partial t} \sigma_{0}+2 \pi i \epsilon^{k} \sigma_{1}+2 \pi i \epsilon^{-k} \sigma_{2}\right) \tilde{\psi}_{A_{\epsilon}^{k}(\mu)},
\end{aligned}
$$

while the action of the $R_{\eta}^{\sigma}$ is by

$$
\tilde{\pi}\left(R_{\eta}^{\sigma}\right) \tilde{\psi}_{\lambda}=\tilde{\psi}_{\lambda+\eta}
$$

As in [2], one can write the operator $\tilde{\not \partial}^{(\mu)}$ as a product

$$
\tilde{\not}^{(\mu)}=D_{\mu} B_{\mu}
$$

with

$$
\begin{aligned}
D_{\mu} \tilde{\psi}_{A_{\epsilon}^{k}(\mu)} & =\operatorname{sign}(N(\mu))|N(\mu)|^{1 / 2} \tilde{\psi}_{A_{\epsilon}^{k}(\mu)}, \\
B_{\mu} \tilde{\psi}_{A_{\epsilon}^{k}(\mu)} & =\left(|N(\mu)|^{-1 / 2} \frac{\partial}{\partial t} \sigma_{0}+2 \pi i \epsilon^{k} \sigma_{1}+2 \pi i \epsilon^{-k} \sigma_{2}\right) \tilde{\psi}_{A_{\epsilon}^{k}(\mu)} .
\end{aligned}
$$

In the following, we relate the Dirac operator $\partial_{X_{\epsilon}}$, its unitarily equivalent operators discussed here above and the decomposition (7.32) to known differential operators on noncommutative tori. 


\subsection{Differential operators on noncommutative tori}

Notice that the action on $\mathbb{R}^{2}$ of the 1-paramater subgroup of $\mathrm{SL}_{2}(\mathbb{R})$

$$
\Theta_{t}=\left(\begin{array}{cc}
e^{t} & 0 \\
0 & e^{-t}
\end{array}\right)
$$

has fixed point $(0,0)$, with stable manifold the axis $(0, y)$ and unstable manifold the axis $(x, 0)$. On the standard torus $\mathbb{R}^{2} / \mathbb{Z}^{2}$ with coordinates $\left(s_{1}, s_{2}\right)$ with $(x, y)=\left(s_{1}+s_{2} \theta, s_{1}+s_{2} \theta^{\prime}\right)$, these two directions define the two Kronecker foliations $s_{1}+s_{2} \theta$ and $s_{1}+s_{2} \theta^{\prime}$ with conjugate slopes $\theta$ and $\theta^{\prime}$. The points of the lattice $\Lambda$ determine on these two foliations the points of the pseudolattices $\mathbb{Z}+\mathbb{Z} \theta$ and $\mathbb{Z}+\mathbb{Z} \theta^{\prime}$, which define the equivalence relation on the space of leaves of the two Kronecker foliations, defining as quotients the noncommutative tori $\mathbb{T}_{\Lambda, i}, i=1,2$. The action of $\Theta_{t}$ is expanding along the line $L_{\theta}=\left\{s_{1}+s_{2} \theta\right\}$ and contracting along $L_{\theta^{\prime}}=\left\{s_{1}+s_{2} \theta^{\prime}\right\}$ and flows the other points of $\mathbb{R}^{2}$ along hyperbola with asymptotes $L_{\theta^{\prime}}$ and $L_{\theta}$.

Thus, the operators $e^{t} \frac{\partial}{\partial x}$ and $e^{-t} \frac{\partial}{\partial y}$ correspond to derivations along the leaf direction of these two transverse Kronecker foliations. The factors $e^{t}$ and $e^{-t}$ are the normalization factors that account for the rescaling of the transverse measure due to the action of the flow $\Theta_{t}$. In fact, consider for instance a small transversal of length $\ell$ for the Kronecker foliation $L_{\theta}$, given by the interval $T_{\ell}=\{(x, y): x=1,-\ell / 2<y<\ell / 2\}$. The flow $\Theta_{t}$ maps it to the transversal $\Theta_{t}\left(T_{\ell}\right)=\left\{\left(x=e^{t}, y\right):-e^{-t} \ell / 2<y<e^{-t} \ell / 2\right\}$ of length $e^{-t} \ell$. Thus, the differentiation $\frac{\partial}{\partial x}$ in the leaf direction of $L_{\theta}$ is weighted by the factor $e^{t}$ that normalizes the length of the transversal and corrects for the scaling of the transverse measure.

Consider then the terms $2 \pi i \lambda_{1} \sigma_{1}$ and $2 \pi i \lambda_{2} \sigma_{2}$ in the operator

$$
\hat{\partial}_{X_{\epsilon}}: \psi_{\lambda} \longmapsto\left(\frac{\partial}{\partial t} \sigma_{0}+2 \pi i \lambda_{1} \sigma_{1}+2 \pi i \lambda_{2} \sigma_{2}\right) \psi_{\lambda},
$$

which we obtained after passing to Fourier modes on the fiber tori $T_{t}^{2}$. These terms correspond, respectively, to the leafwise derivations $e^{t} \frac{\partial}{\partial x}$ and $e^{-t} \frac{\partial}{\partial y}$. These can be expressed equivalently in terms of the operators

$$
\delta_{\theta}: \psi_{n, m} \longmapsto(n+m \theta) \psi_{n, m} \quad \text { and } \quad \delta_{\theta^{\prime}}: \psi_{n, m} \longmapsto\left(n+m \theta^{\prime}\right) \psi_{n, m},
$$

so that the sum $\lambda_{1} \sigma_{1}+\lambda_{2} \sigma_{2}$ acts as the operator

$$
\not D_{\theta, \theta^{\prime}}=\left(\begin{array}{cc}
0 & \delta_{\theta^{\prime}}-i \delta_{\theta} \\
\delta_{\theta^{\prime}}+i \delta_{\theta} & 0
\end{array}\right) .
$$


This gives the Dirac operator of a spectral triple on the noncommutative tori $\mathbb{T}_{\Lambda, i}$ with

$$
R_{r, k}^{\sigma} \psi_{n, m}=\sigma((r, k),(n, m)) \psi_{(n, m)+(r, k)}
$$

and

$$
\left[\not D_{\theta, \theta^{\prime}}, R_{r, k}^{\sigma}\right]=\left(\begin{array}{cc}
0 & \left(r+k \theta^{\prime}\right)-i(r+k \theta) \\
\left(r+k \theta^{\prime}\right)+i(r+k \theta) & 0
\end{array}\right) R_{r, k}^{\sigma}
$$

In the particular case where $\theta^{\prime}=-\theta$, this agrees with the spectral triple for the first order signature operator on the noncommutative torus considered, for instance, in [22]. The construction of [22] can be interpreted as obtained by using the two transverse Kronecker foliations $L_{\theta}$ and $L_{-\theta}$ and the associated leafwise derivations $\partial / \partial x$ and $\partial / \partial y$.

We can consider here the same kind of unitary transformations that we described earlier for $\hat{\partial}_{X_{\epsilon}}$, applied to the operator $\not_{\theta, \theta^{\prime}}$ of (7.33). Let us denote by $\not_{\theta, \theta^{\prime}, 0}$ the restriction of $\not_{\theta, \theta^{\prime}}$ to the complement of the zero modes $\psi_{0}$ (i.e., $\left.\lambda=0\right)$. We have, as in (7.30),

$$
\not D_{\theta, \theta^{\prime}, 0}=\sum_{\mu \in(\Lambda \backslash\{0\}) / V} \not D_{\theta, \theta^{\prime}}^{\mu}
$$

with

$$
\not D_{\theta, \theta^{\prime}}^{\mu} \psi_{A_{\epsilon}^{k}(\mu)}=\left(\lambda_{1} \sigma_{1}+\lambda_{2} \sigma_{2}\right) \psi_{A_{\epsilon}^{k}(\mu)} .
$$

After the unitary transformation $\tilde{\mathcal{U}} \mathcal{U}$ with $\mathcal{U}$ as in (7.25) and $\tilde{\mathcal{U}}$ as in (7.29), we obtain a unitarily equivalent operator

$$
\tilde{D}_{\theta, \theta^{\prime}}^{\mu} \tilde{\psi}_{A_{\epsilon}^{k}(\mu)}=\operatorname{sign}(N(\mu))|N(\mu)|^{1 / 2}\left(\epsilon^{k} \sigma_{1}+\epsilon^{-k} \sigma_{2}\right) \tilde{\psi}_{A_{\epsilon}^{k}(\mu)}
$$

As before, we factor this as a product of the operators

$$
\tilde{D}_{\theta, \theta^{\prime}}^{\mu}=D_{\theta}^{\mu} B_{\theta}
$$

with

$$
\begin{aligned}
& D_{\theta}^{\mu} \tilde{\psi}_{A_{\epsilon}^{k}(\mu)}=\operatorname{sign}(N(\mu))|N(\mu)|^{1 / 2} \tilde{\psi}_{A_{\epsilon}^{k}(\mu)}, \\
& B_{\theta} \tilde{\psi}_{A_{\epsilon}^{k}(\mu)}=\left(\epsilon^{k} \sigma_{1}+\epsilon^{-k} \sigma_{2}\right) \tilde{\psi}_{A_{\epsilon}^{k}(\mu)} .
\end{aligned}
$$

\section{Shimizu $L$-function and Lorentzian geometry}

In this section, we describe another way of relating the Shimizu $L$-function to the geometry of noncommutative tori with real multiplication, by regarding 
the norms $N(\lambda)$, for $\lambda \in \Lambda$, as defining the momenta of a Lorentzian rather than Euclidean Dirac operator.

Instead of working with positive inner product spaces, as in the case of Euclidean spectral triples, the Galois involution of the real quadratic field defines a natural choice of a "Krein involution" and the norm correspondingly defines an indefinite quadratic form. One formulates in this way a notion of spectral triple over a real quadratic field and with Lorentzian signature, using the relation between indefinite inner product spaces and the associated real Hilbert spaces. The main point that requires care is the fact that the Lorentzian Dirac operator has a noncompact group of symmetries, in our case given by the units of the real quadratic field, hence it fails to have compact resolvent due to the presence of infinite multiplicities in the eigenvalues. We show that the multiplicities can be resolved by transforming the triple via a Krein isometry, which is an unbounded self-adjoint operator in the associated real Hilbert space and defines a finitely summable associated Dirac operator in the Euclidean signature.

As above, we let $\Lambda$ be the lattice in $\mathbb{R}^{2}$ associated to a lattice $L \subset \mathbb{K}$ in a real quadratic field $\mathbb{K}=\mathbb{Q}(\sqrt{d})$ by the embeddings $\iota_{i}: \mathbb{K} \hookrightarrow \mathbb{R}$,

$$
\Lambda=\left\{\lambda \in \mathbb{R}^{2} \mid \lambda=\left(\lambda_{1}, \lambda_{2}\right)=\left(\iota_{1}(\ell), \iota_{2}(\ell)\right), \ell \in L\right\} .
$$

We denote, as above, by $V$ the group $V=\epsilon^{\mathbb{Z}}$ of units preserving $\Lambda$. We denote the action as above with $\lambda \mapsto A_{\epsilon}^{k}(\lambda)=\left(\epsilon^{k} \lambda_{1}, \epsilon^{-k} \lambda_{2}\right)$.

For $x \in \mathbb{K}$, we denote by $x^{\prime}=c(x)$ the image under the Galois involution of $\mathbb{K}$. For $\lambda=\left(\lambda_{1}, \lambda_{2}\right) \in \Lambda$, we have $\lambda_{2}=c\left(\lambda_{1}\right)$. The norm is given by $N(\lambda)=\lambda_{1} \lambda_{2}$, and $N(\epsilon)=\epsilon \epsilon^{\prime}=1$.

We consider the quadratic form $N(\lambda)=\lambda_{1} \lambda_{2}=(n+m \theta)\left(n+m \theta^{\prime}\right)$ to be the analog of the wave operator $\square=p_{0}^{2}-p_{1}^{2}$.

Its Dirac factorization into linear first order operator is obtained by considering a linear operator of the form

$$
\mathcal{D}_{\lambda}=\left(\begin{array}{cc}
0 & \mathcal{D}_{\lambda}^{+} \\
\mathcal{D}_{\lambda}^{-} & 0
\end{array}\right):=\left(\begin{array}{cc}
0 & \lambda_{1} \\
\lambda_{2} & 0
\end{array}\right)
$$

whose square is $\mathcal{D}_{\lambda}^{2}=\square_{\lambda}$, with

$$
\square_{\lambda}=\left(\begin{array}{cc}
N(\lambda) & 0 \\
0 & N(\lambda)
\end{array}\right) .
$$

We assemble these modes to define an operator $\mathcal{D}$ acting on $\mathcal{H}=\ell^{2}(\Lambda) \oplus$ $\ell^{2}(\Lambda)$ by $\mathcal{D} e_{\lambda, \pm}=\mathcal{D}_{\lambda} e_{\lambda, \pm}$. This satisfies $\mathcal{D} \gamma=-\gamma \mathcal{D}$ with respect to the 
$\mathbb{Z} / 2 \mathbb{Z}$-grading

$$
\gamma=\left(\begin{array}{cc}
1 & 0 \\
0 & -1
\end{array}\right)
$$

Consider the algebra $C^{*}(\Lambda, \sigma)$ of the noncommutative torus acting diagonally on $\mathcal{H}$. The operator $\mathcal{D}$ has bounded commutators with the elements of the dense subalgebra $\mathbb{C}(\Lambda, \sigma)$ since we have

$$
\left[\mathcal{D}, R_{\eta}^{\sigma}\right] e_{\lambda, \pm}=\sigma(\lambda, \eta) \eta_{\mp} e_{\eta+\lambda, \pm},
$$

where we used the notation $\eta_{+}=\eta_{1}$ and $\eta_{-}=\eta_{2}$.

However, the other properties of $\mathcal{D}$ differ significantly from what one usually postulates for Dirac operators of spectral triples.

First of all, notice that $\mathcal{D}$ is not self-adjoint. In fact, it is invariant with respect to a different involution, defined for operators with coefficients in the real quadratic field $\mathbb{K}$, namely

$$
\mathcal{D}=c\left(\mathcal{D}^{t}\right),
$$

where $\mathcal{D}^{t}=\left(\mathcal{D}_{\lambda}^{t}\right)$ denotes the transpose and $c(\mathcal{D})$ denotes the effect of the Galois involution $c: x \mapsto x^{\prime}$ of $\mathbb{K}$ applied to the coefficients of $\mathcal{D}$. In this arithmetic context, it is natural to require this property instead of selfadjointness.

A more serious problem, however, comes from the fact that the operator $\mathcal{D}$ has infinite multiplicities, hence it is very far from having the compact resolvent property of spectral triple. This is a typical problem one encounters in trying to extend the formalism of spectral triples from the Euclidean to the Lorentzian context, because of the presence of noncompact symmetry groups for the Lorentzian manifold. Here the noncompact symmetry group is given by the units in $V=\epsilon^{\mathbb{Z}}$.

\subsection{Arithmetic Krein spaces}

It is well known that, when one replaces Euclidean geometry by Lorentzian geometry, the notion of the Hilbert space is replaced by the notion of a Krein space (cf. e.g., [7]). The version we consider here is slightly different from the usual one, since we want to be able to work over the real quadratic field $\mathbb{K}$ instead of passing directly to complex numbers.

Definition 8.1. Let $c: \mathbb{K} \rightarrow \mathbb{K}$ denote the Galois involution $c: x \mapsto x^{\prime}$ of the real quadratic field. Let $\mathcal{V}$ be a $\mathbb{K}$-vector space. We say that a map $T: \mathcal{V} \rightarrow \mathcal{V}$ is $c$-linear if it satisfies $T(a v+b w)=c(a) T(v)+c(b) T(w)$. 
A Lorentzian pairing on a $\mathbb{K}$-vector space $\mathcal{V}$ is a nondegenerate $\mathbb{K}$-valued pairing

$$
(\cdot, \cdot): \mathcal{V} \times \mathcal{V} \longrightarrow \mathbb{K}
$$

which is $c$-linear in the first variable and linear in the second, i.e., it is conjugate-symmetric with respect to the Galois involution $c$.

We can then introduce the analog of the notion of a Krein space in this arithmetic context.

Definition 8.2. A Krein space over a real quadratic field $\mathbb{K}$ (or $\mathbb{K}$-Krein space) is a $\mathbb{K}$-vector space $\mathcal{V}$ endowed with a Lorentzian pairing $(\cdot, \cdot)$ as in Definition 8.1, and a $c$-linear involution $\kappa: \mathcal{V} \rightarrow \mathcal{V}$, such that the pairing $(\kappa \cdot, \cdot)$ has the following properties:

(1) $(\kappa \cdot, \cdot)=c(\cdot, \kappa \cdot)$;

(2) For all $v \neq 0$ in $\mathcal{V}$, the elements $(\kappa v, v) \in \mathbb{K}$ are totally positive.

When properties (1) and (2) of Definition 8.2 hold, we say that $(\kappa \cdot, \cdot)$ defines a positive-definite inner product. We have a corresponding notion of Krein adjoint as follows.

Definition 8.3. Given a $\mathbb{K}$-linear operator $T$ on a $\mathbb{K}$-Krein space $\mathcal{V}$, the Krein adjiont $T^{\dagger}$ is the adjoint in the Lorentzian pairing $(\cdot, \cdot)$,

$$
(v, T w)=\left(T^{\dagger} v, w\right)
$$

The $c$-linear involution $\kappa$ of Definition 8.2 corresponds to a Wick rotation from Lorentzian to Euclidean signature. The Krein adjoint satisfies $T^{\dagger}=$ $\kappa T^{*} \kappa$, where $T^{*}$ is the adjoint in the inner product $\langle\cdot, \cdot\rangle=(\kappa \cdot, \cdot)$.

Given a $\mathbb{K}$-Krein space $\mathcal{V}$, there are two naturally associated real Hilbert spaces, obtained by considering the real vector spaces

$$
\mathcal{V}_{\mathbb{R}, i}:=\mathcal{V} \otimes_{\iota_{i}(\mathbb{K})} \mathbb{R}
$$

obtained by tensoring $\mathcal{V}$ with $\mathbb{R}$ using either one of the two embeddings $\iota_{i}: \mathbb{K} \hookrightarrow \mathbb{R}$ of the real quadratic field. 
Lemma 8.4. The pairing

$$
\langle v, w\rangle=\frac{1}{2} \iota_{1}((\kappa v, w)+(v, \kappa w))=\frac{1}{2} \iota_{2}((\kappa v, w)+(v, \kappa w))
$$

induced on $\mathcal{V}_{\mathbb{R}, i}$ by the Lorentzian pairing $(\cdot, \cdot)$ on $\mathcal{V}$ defines a real valued positive-definite inner product.

Proof. We know that $(v, \kappa w)=c(\kappa v, w)$. Thus, we have

$$
\langle v, w\rangle=\frac{1}{2}\left(\iota_{1}(\kappa v, w)+\iota_{2}(\kappa v, w)\right) .
$$

We can extend this pairing by $\mathbb{R}$-linearity to define a bilinear form on $\mathcal{V}_{\mathbb{R}, i}$. By the assumption that for $v \neq 0$ the $(\kappa v, v)$ are totally positive elements of $\mathbb{K}$, we obtain that (8.7) defines a positive-definite inner product.

In the following, we still denote by $\mathcal{V}_{\mathbb{R}, i}$ the Hilbert space completion obtained in this way.

\subsection{Lorentzian spectral triples over real quadratic fields}

It is not uncommon to make use of Krein spaces to extend the formalism of spectral triples to Lorentzian geometry [29]. Here we follow a similar viewpoint, adapted to the arithmetic setting of real quadratic fields.

For a $\mathbb{K}$-linear operator $T$ acting on a $\mathbb{K}$-Krein space $\mathcal{V}$, we define $\mathbb{M}_{i}(T) \geq-\infty$, for $i=1,2$, as

$$
\mathbb{M}_{i}(T):=\inf _{(v, v)=1} \iota_{i}(T v, T v)
$$

We introduce the following preliminary notion of a $\mathbb{K}$-triple, which we then refine by additional properties providing the analog of a spectral triple.

Definition 8.5. A Krein $\mathbb{K}$-triple consists of data $(\mathcal{A}, \mathcal{V}, \mathcal{D})$ with the following properties.

(1) $\mathcal{A}$ is an involutive algebra over the real quadratic field $\mathbb{K}$.

(2) $\mathcal{V}$ is a $\mathbb{K}$-Krein space with nondegenerate $\mathbb{K}$-bilinear form $(\cdot, \cdot)$.

(3) The algebra $\mathcal{A}$ acts on $\mathcal{V}$ via a representation $\pi: \mathcal{A} \rightarrow \operatorname{End}_{\mathbb{K}}(\mathcal{V})$, with the involution of $\mathcal{A}$ realized by the Krein adjoint $\pi\left(a^{*}\right)=\pi(a)^{\dagger}$. 
(4) The operators $\pi(a)$, for $a \in \mathcal{A}$, satisfy

$$
\mathbb{M}_{i}(a)>-\infty
$$

(5) The operator $\mathcal{D}$ is a densely defined $\mathbb{K}$-linear operator on $\mathcal{V}$, which is Krein-self-adjoint , $\mathcal{D}^{\dagger}=\mathcal{D}$.

(6) The commutators $C_{a}:=[\mathcal{D}, a]$ satisfy

$$
\mathbb{M}_{i}\left(C_{a}\right)>-\infty, \quad \forall a \in \mathcal{A}
$$

We then define Lorentzian $\mathbb{K}$-spectral triples in the following way.

Definition 8.6. A Krein $\mathbb{K}$-triple $(\mathcal{A}, \mathcal{V}, \mathcal{D})$ as in Definition 8.5 is a finitely summable Lorentzian $\mathbb{K}$-spectral triple if the following holds.

(1) There exists a densely defined $\mathbb{K}$-linear operator $U: \mathcal{V} \rightarrow \mathcal{V}$ with $(U v$, $U v)=(v, v)$, for all $v \in \operatorname{Dom}(U)$ and $U^{\dagger}=U^{-1}$, with the property that

$$
U^{\dagger} \mathcal{D} U=\mathcal{D}
$$

(2) The commutators $C_{a, U}:=\left[\mathcal{D}_{U}, \pi_{U}(a)\right]$, with $\pi_{U}(a)=U^{\dagger} \pi(a) U$, satisfy the condition

$$
\mathbb{M}_{i}\left(C_{a, U}\right)>-\infty, \quad \forall a \in \mathcal{A}
$$

(3) The operator $U$ is an unbounded self-adjoint operator, $U=U^{*}$, on the associated real Hilbert space $\mathcal{V}_{\mathbb{R}, i}$ with the inner product $\langle\cdot, \cdot\rangle$ of $(8.7)$.

(4) The triple $(\mathcal{A}, \mathcal{V}, \mathcal{D})$ is $p$-summable for $p \in \mathbb{R}_{+}^{*}$ if

$$
\sum_{n}\left|\left\langle U e_{n},\left|\mathcal{D}^{2}\right| U e_{n}\right\rangle\right|^{-s / 2}<\infty, \quad \forall s \geq p,
$$

where $e_{n}$ is an orthonormal basis for the complement of the zero modes of the operator $\left|\mathcal{D}^{2}\right|$ in the real Hilbert space $\mathcal{V}_{\mathbb{R}, i}$.

Notice that in Krein spaces isometries are not necessarily bounded operators [7, Section VI], so the $U$ is only densely defined in general. The definition given here is different from the notions of Lorentzian spectral triples currently developed in the literature. The differences stem mainly from our need to work over a finite extension of $\mathbb{Q}$ instead of $\mathbb{C}$ and to resolve the 
infinite multiplicity of the eigenvalues. We also require the weaker property (8.9), (8.10) and (8.12), instead of requiring continuity in the operator norm in the associated Hilbert space. These conditions will become more transparent in our main example below.

\subsection{Arithmetic twisted group algebras}

We consider the $\mathbb{K}$-vector space $\mathcal{V}_{\Lambda}$ spanned by the basis elements $e_{\lambda}$ with $\lambda \in \Lambda$, endowed with the pairing

$$
(v, w):=\sum_{\lambda} c\left(a_{\lambda}\right) b_{\lambda}
$$

for $v=\sum_{\lambda} a_{\lambda} e_{\lambda}$ and $w=\sum_{\lambda} b_{\lambda} e_{\lambda}$, and with $c: x \mapsto x^{\prime}$ the Galois involution of $\mathbb{K}$.

Lemma 8.7. The space $\mathcal{V}_{\Lambda}$ with the pairing (8.14) is a $\mathbb{K}$-Krein space.

Proof. Clearly the pairing (8.14) is a Lorentzian pairing in the sense of Definition 8.1. Let $\kappa: \mathcal{V}_{\Lambda} \rightarrow \mathcal{V}_{\Lambda}$ be given by the Galois involution

$$
\kappa(v)=\sum_{\lambda} c\left(a_{\lambda}\right) e_{\lambda}
$$

Then the pairing $\langle v, w\rangle=(\kappa v, w)=c(v, \kappa w)$ is a positive-definite inner product, as in Definition 8.2. In fact, we have

$$
\iota_{1}\langle v, v\rangle=\sum_{\lambda} \iota_{1}\left(a_{\lambda}^{2}\right) \geq 0, \quad \iota_{2}\langle v, v\rangle=\sum_{\lambda} \iota_{2}\left(a_{\lambda}^{2}\right) \geq 0
$$

We consider on $\mathcal{V}_{\Lambda}$ the action of the group ring $\mathbb{K}[\Lambda]$, given by $R_{\lambda} e_{\eta}=$ $e_{\lambda+\eta}$

Lemma 8.8. The operators $R_{\lambda}$ acting on $\mathcal{V}_{\Lambda}$ satisfy

$$
\mathbb{M}_{i}\left(R_{\lambda}\right)>-\infty
$$

Moreover, the operators $R_{\lambda}$ define bounded operators in the associated real Hilbert spaces $\mathcal{V}_{\Lambda, \mathbb{R}, i}=\mathcal{V}_{\Lambda} \otimes_{\iota_{i}(\mathbb{K})} \mathbb{R}$. 
Proof. The operators $R_{\lambda}$ are Krein isometries, and $\left(R_{\lambda} v, R_{\lambda} v\right)=(v, v)$ implies that $\mathbb{M}_{i}\left(R_{\lambda}\right)=1$. The operators $R_{\lambda}$ act by $e_{\eta} \mapsto e_{\lambda+\eta}$ on the associated Hilbert spaces, hence they define bounded (unitary) operators.

Now we want to introduce, in this setting of $\mathbb{K}$-Krein spaces, an analog of the twisted group ring $\mathbb{C}(\Lambda, \sigma)$ (the noncommutative torus) we have been working with in the complex case.

Lemma 8.9. Suppose given $\omega \in \mathbb{K}^{*}$ with $N(\omega)=\omega \omega^{\prime}=1$. Then the expression

$$
\varpi(\lambda, \eta)=\omega^{(n, m) \wedge(r, k)},
$$

for $\lambda=\left(n+m \theta, n+m \theta^{\prime}\right)$ and $\eta=\left(r+k \theta, r+k \theta^{\prime}\right)$, defines a $\mathbb{K}^{*}$-valued group 2-cocycle $\sigma$ on $\Lambda$.

Proof. The argument is the same as in the complex case. It suffices to show that the cocycle condition holds.

Definition 8.10. The twisted group ring $\mathbb{K}(\Lambda, \sigma)$ is the unital involutive $\mathbb{K}$-algebra generated by elements $R_{\lambda}^{\varpi}$ with the product

$$
R_{\lambda}^{\varpi} R_{\eta}^{\varpi}=\varpi(\lambda, \eta) R_{\lambda+\eta}^{\varpi}=\omega^{(n, m) \wedge(r, k)} R_{\lambda+\eta}^{\varpi},
$$

for $\lambda=\left(n+m \theta, n+m \theta^{\prime}\right)$ and $\eta=\left(r+k \theta, r+k \theta^{\prime}\right)$, and the involution $\left(R_{\lambda}^{\varpi}\right)^{*}=R_{-\lambda}^{\varpi}$.

The twisted group ring $\mathbb{K}(\Lambda, \sigma)$ also acts on $\mathcal{V}_{\Lambda}$ by

$$
R_{\lambda}^{\varpi} e_{\eta}=\varpi(\eta, \lambda) e_{\lambda+\eta} .
$$

Lemma 8.11. The operators $R_{\lambda}^{\varpi}$, acting as in (8.17), satisfy $\mathbb{M}_{i}\left(R_{\lambda}^{\varpi}\right)>-\infty$.

Proof. The action (8.17) preserves the Lorentzian pairing $(\cdot, \cdot)$ on $\mathcal{V}_{\Lambda}$ since

$$
\left(R_{\lambda}^{\varpi} e_{\eta}, R_{\lambda}^{\varpi} e_{\zeta}\right)=c(\omega(\eta, \lambda)) \omega(\zeta, \lambda) \delta_{\eta, \zeta}=N(\omega(\eta, \lambda)) \delta_{\eta, \zeta}=\left(e_{\eta}, e_{\zeta}\right),
$$

since $N(\omega(\eta, \lambda))=1$. The condition $\left(R^{\varpi} v, R^{\varpi} v\right)=(v, v)$ implies $\mathbb{M}_{i}\left(R_{\lambda}^{\varpi}\right)=1$.

However, notice that, while the action of $\mathbb{K}[\Lambda]$ extends to an action by bounded operators on the associated real Hilbert spaces $\mathcal{V}_{\mathbb{K}} \otimes_{\iota_{i}(\mathbb{K})} \mathbb{R}$, the 
induced action on $\mathcal{V}_{\mathbb{R}}$ of the twisted group ring $\mathbb{K}(\Lambda, \varpi)$ is by the unbounded operators

$$
R_{\lambda}^{\varpi} e_{\eta, \pm}=A_{\omega}^{(r, k) \wedge(n, m)} e_{\lambda+\eta, \pm}
$$

with

$$
A_{\omega}=\left(\begin{array}{cc}
\iota_{1}(\omega) & 0 \\
0 & \iota_{2}(\omega)
\end{array}\right) \in \mathrm{SL}_{2}(\mathbb{R})
$$

As in the complex case, we can also consider the group ring $\mathbb{K}[S(\Lambda, V)]$ for $S(\Lambda, V)=\Lambda \rtimes V$. The cocycle (8.15) extends to a cocycle on the cross product by setting

$$
\tilde{\varpi}((\lambda, k),(\eta, r))=\varpi\left(\lambda, A_{\epsilon}^{k}(\eta)\right)=\omega^{(n, m) \wedge(u, v) \varphi_{\epsilon}^{k}},
$$

for $\lambda=\left(n+m \theta, n+m \theta^{\prime}\right)$ and $\eta=\left(u+v \theta, u+v \theta^{\prime}\right)$, with $n, m, u, v \in \mathbb{Z}$.

Definition 8.12. The twisted group ring $\mathbb{K}(S(\Lambda, V), \tilde{\varpi})$ is the unitary involutive $\mathbb{K}$-algebra with generators $R_{\lambda, k}^{\tilde{\varpi}}$ satisfying

$$
R_{\lambda, k}^{\tilde{\varpi}} R_{\eta, r}^{\tilde{\varpi}}=\tilde{\varpi}((\lambda, k),(\eta, r)) R_{\lambda+A_{\epsilon}^{k}(\eta), k+r}^{\tilde{\varpi}},
$$

with the involution $\left(R_{\lambda, k}^{\tilde{\tilde{\varpi}}}\right)^{*}=R_{-A_{\epsilon}^{-k}(\lambda),-k}^{\tilde{\varpi}}$.

\subsection{Lorentzian Dirac operator}

On the $\mathbb{K}$-Krein space $\mathcal{V}_{\Lambda} \oplus \mathcal{V}_{\Lambda}$ we consider the densely defined $\mathbb{K}$-linear operator

$$
\mathcal{D}_{\mathbb{K}} e_{\lambda, \pm}=\mathcal{D}_{\mathbb{K}, \lambda} e_{\lambda, \pm}=\left(\begin{array}{cc}
0 & \mathcal{D}_{\lambda}^{+} \\
\mathcal{D}_{\lambda}^{-} & 0
\end{array}\right) e_{\lambda, \pm}:=\left(\begin{array}{cc}
0 & \ell \\
c(\ell) & 0
\end{array}\right) e_{\lambda, \pm}
$$

where we write $\lambda \in \Lambda$ as $\lambda=\left(\iota_{1}(\ell), \iota_{2}(\ell)\right)$ with $\ell \in L \subset \mathbb{K}$, as in (8.1).

The operator $\mathcal{D}_{\mathbb{K}}$ of (8.21) induces on the real Hilbert space $\mathcal{V}_{\mathbb{R}, i} \oplus \mathcal{V}_{\mathbb{R}, i}$ the $\mathbb{R}$-linear operators

$$
\mathcal{D}_{\lambda}=\left(\begin{array}{cc}
0 & \lambda_{1} \\
\lambda_{2} & 0
\end{array}\right) \quad \text { and } \quad c\left(\mathcal{D}_{\lambda}\right)=\left(\begin{array}{cc}
0 & \lambda_{2} \\
\lambda_{1} & 0
\end{array}\right)
$$

respectively. This recovers the Lorentzian Dirac operator described in (8.2) above. 
Lemma 8.13. The data $\left(\mathbb{K}(\Lambda, \varpi), \mathcal{V}_{\Lambda} \oplus \mathcal{V}_{\Lambda}, \mathcal{D}_{\mathbb{K}}\right)$ define a Krein $\mathbb{K}$-triple in the sense of Definition 8.5.

Proof. Properties (1)-(4) of Definition 8.5 follow from Lemma 8.7, Lemma 8.11 and the fact that the Krein adjoint $\left(R_{\lambda}^{\varpi}\right)^{\dagger}=R_{-\lambda}^{\varpi}=\left(R_{\lambda}^{\varpi}\right)^{-1}$. Property (5) follows directly from (8.21), since

$$
\mathcal{D}_{\mathbb{K}}^{\dagger}=c\left(\mathcal{D}_{\mathbb{K}, \lambda}^{t}\right)=\left(\begin{array}{cc}
0 & c\left(\lambda_{2}\right) \\
c\left(\lambda_{1}\right) & 0
\end{array}\right)=\left(\begin{array}{cc}
0 & \lambda_{1} \\
\lambda_{2} & 0
\end{array}\right)=\mathcal{D}_{\mathbb{K}} .
$$

We then need to prove (6), namely that the commutators $\left[\mathcal{D}_{\mathbb{K}}, R_{\lambda}^{\varpi}\right]$ satisfy

$$
\mathbb{M}_{i}\left(\left[\mathcal{D}_{\mathbb{K}}, R_{\lambda}^{\varpi}\right]\right)>-\infty .
$$

We have

$$
\left[\mathcal{D}_{\mathbb{K}}, R_{\lambda}^{\varpi}\right] e_{\eta, \pm}=\varpi(\eta, \lambda)\left(\begin{array}{cc}
0 & \left(\lambda_{1}+\eta_{1}\right)-\eta_{1} \\
\left(\lambda_{2}+\eta_{2}\right)-\eta_{2} & 0
\end{array}\right) e_{\lambda+\eta, \pm} .
$$

Thus, we have

$$
\left(\left[\mathcal{D}_{\mathbb{K}}, R_{\lambda}^{\varpi}\right] v,\left[\mathcal{D}_{\mathbb{K}}, R_{\lambda}^{\varpi}\right] v\right)=N(\lambda)(v, v),
$$

from which the result follows.

Suppose given a choice of a fundamental domain $\mathcal{F}_{V}$ for the action of $V=\epsilon^{\mathbb{Z}}$ on $\Lambda$. Let $\rho(\lambda) \in \mathbb{Z}$ denote the unique integer such that $\lambda=A_{\epsilon}^{\rho(\lambda)}(\mu)$, with $\mu \in \mathcal{F}_{V}$.

Consider the $\mathbb{K}$-linear operator on $\mathcal{V}_{\Lambda} \oplus \mathcal{V}_{\Lambda}$ defined by

$$
T_{\epsilon} e_{\lambda, \pm}:=\left(\begin{array}{cc}
\epsilon^{\rho(\lambda)} & 0 \\
0 & \epsilon^{-\rho(\lambda)}
\end{array}\right) e_{\lambda, \pm} .
$$

Consider also the involution $J: \mathcal{V} \rightarrow \mathcal{V}$ defined by setting

$$
J e_{\lambda, \pm}=e_{J(\lambda), \pm}
$$

where $J(\lambda)=A_{\epsilon}^{-k}(\mu)$ for $\lambda=A_{\epsilon}^{k}(\mu)$ with $\mu \in \mathcal{F}_{V}$ and $k \in \mathbb{Z}$. This satisfies $J^{2}=1$ and $J^{\dagger}=J$.

We set $U_{\epsilon}=T_{\epsilon} J$, with

$$
U_{\epsilon} e_{\lambda, \pm}=\left(\begin{array}{cc}
\epsilon^{-\rho(\lambda)} & 0 \\
0 & \epsilon^{\rho(\lambda)}
\end{array}\right) e_{J(\lambda), \pm}
$$

We now show that the data of Lemma 8.13 satisfy the properties a Lorentzian $\mathbb{K}$-spectral triple. 
Proposition 8.14. The data $\left(\mathbb{K}(\Lambda, \varpi), \mathcal{V}_{\Lambda} \oplus \mathcal{V}_{\Lambda}, \mathcal{D}_{\mathbb{K}}\right)$ define a Lorentzian $\mathbb{K}$-spectral triple, as in Definition 8.6.

Proof. The $T_{\epsilon}$ are Krein isometries, since

$$
\left(T_{\epsilon} e_{\lambda, \pm}, T_{\epsilon} e_{\lambda, \pm}\right)=N\left(\epsilon^{\rho(\lambda)}\right)\left(e_{\lambda, \pm}, e_{\lambda, \pm}\right)=\left(e_{\lambda, \pm}, e_{\lambda, \pm}\right)
$$

They satisfy $T_{\epsilon}^{\dagger}=T_{\epsilon^{-1}}=T_{\epsilon}^{-1}$. Thus we have $U_{\epsilon}^{\dagger}=J^{\dagger} T_{\epsilon}^{\dagger}=J T_{\epsilon}^{-1}=U_{\epsilon}^{-1}$. This is also a Krein isometry since both $T_{\epsilon}$ and $J$ are, with $\operatorname{Dom}\left(U_{\epsilon}\right)=$ $\operatorname{Dom}\left(T_{\epsilon}\right)$, since $J$ is bounded.

The operator $U_{\epsilon}$ is a symmetry of the Dirac operator, namely we have

$$
\mathcal{D}_{\mathbb{K}, \epsilon}:=U_{\epsilon}^{\dagger} \mathcal{D}_{\mathbb{K}} U_{\epsilon}=\mathcal{D}_{\mathbb{K}}
$$

In fact, we have

$$
T_{\epsilon}^{\dagger} \mathcal{D}_{\mathbb{K}} T_{\epsilon} e_{\lambda, \pm}=\left(\begin{array}{cc}
0 & \epsilon^{-2 \rho(\lambda)} \lambda_{1} \\
\epsilon^{2 \rho(\lambda)} \lambda_{2} & 0
\end{array}\right) e_{\lambda, \pm}
$$

Since we have $\lambda=A_{\epsilon}^{\rho(\lambda)}(\mu)$ with $\mu \in \mathcal{F}_{V}$, we can write the above equivalently as

$$
T_{\epsilon}^{\dagger} \mathcal{D}_{\mathbb{K}} T_{\epsilon} e_{\lambda, \pm}=\left(\begin{array}{cc}
0 & \epsilon^{-\rho(\lambda)} \mu_{1} \\
\epsilon^{\rho(\lambda)} \mu_{2} & 0
\end{array}\right) e_{\lambda, \pm}
$$

Thus, we have

$$
\begin{aligned}
J^{\dagger} T_{\epsilon}^{\dagger} \mathcal{D}_{\mathbb{K}} T_{\epsilon} J e_{\lambda, \pm} & =\left(\begin{array}{cc}
0 & \epsilon^{\rho(\lambda)} \mu_{1} \\
\epsilon^{-\rho(\lambda)} \mu_{2} & 0
\end{array}\right) e_{\lambda, \pm} \\
& =\left(\begin{array}{cc}
0 & \lambda_{1} \\
\lambda_{2} & 0
\end{array}\right) e_{\lambda, \pm}=\mathcal{D}_{\mathbb{K}} e_{\lambda, \pm}
\end{aligned}
$$

This proves property (1) of Definition 8.6. We now show that (2) and (3) of Definition 8.6 also hold.

(2) follows from Lemma 8.13 and the fact that $U_{\epsilon}$ is a Krein isometry, since

$$
\begin{aligned}
\left(U_{\epsilon}^{\dagger}\left[\mathcal{D}_{\mathbb{K}}, R_{\lambda}^{\varpi}\right] U_{\epsilon} v, U_{\epsilon}^{\dagger}\left[\mathcal{D}_{\mathbb{K}}, R_{\lambda}^{\varpi}\right] U_{\epsilon} v\right) & =\left(\left[\mathcal{D}_{\mathbb{K}}, R_{\lambda}^{\varpi}\right] U_{\epsilon} v,\left[\mathcal{D}_{\mathbb{K}}, R_{\lambda}^{\varpi}\right] U_{\epsilon} v\right) \\
& =N(\lambda)\left(U_{\epsilon} v, U_{\epsilon} v\right)=N(\lambda)(v, v)
\end{aligned}
$$


(3) The adjoint $U_{\epsilon}^{*}$ in the associated Hilbert space inner product $\langle\cdot, \cdot\rangle$ of (8.7) satisfies $U_{\epsilon}^{*}=U_{\epsilon}$ since

$$
\begin{aligned}
U_{\epsilon}^{*} e_{\lambda, \pm} & =\left(\kappa U_{\epsilon}^{\dagger} \kappa\right) e_{\lambda, \pm}=c\left(U_{\epsilon}^{\dagger}\right) e_{\lambda, \pm} \\
& =\left(\begin{array}{cc}
c\left(\epsilon^{\rho(\lambda)}\right) & 0 \\
0 & c\left(\epsilon^{-\rho(\lambda)}\right)
\end{array}\right) e_{J(\lambda), \pm}=\left(\begin{array}{cc}
\epsilon^{-\rho(\lambda)} & 0 \\
0 & \epsilon^{\rho(\lambda)}
\end{array}\right) e_{J(\lambda), \pm}=U_{\epsilon} e_{\lambda, \pm} .
\end{aligned}
$$

Consider then the operator $\left|\mathcal{D}_{\mathbb{K}}^{2}\right|$ acting on the associated real Hilbert space by

$$
\left|\mathcal{D}_{\mathbb{K}}^{2}\right| e_{\lambda, \pm}=\left(\begin{array}{cc}
|N(\lambda)| & 0 \\
0 & |N(\lambda)|
\end{array}\right) e_{\lambda, \pm}
$$

We restrict $\left|\mathcal{D}_{\mathbb{K}}^{2}\right|$ to the orthogonal complement of the zero modes, i.e., on the span of the $e_{\lambda, \pm}$ with $\lambda \neq 0$. We then obtain

$$
\sum_{\lambda \neq 0}\left|\left\langle U_{\epsilon} e_{\lambda, \pm},\left|\mathcal{D}_{\mathbb{K}}^{2}\right| U_{\epsilon} e_{\lambda, \pm}\right\rangle\right|^{-s / 2}=\sum_{\lambda \neq 0}\left(\epsilon^{2 \rho(\lambda)}+\epsilon^{-2 \rho(\lambda)}\right)^{-s / 2}|N(\lambda)|^{-s / 2} .
$$

This can be written equivalently as

$$
\sum_{k \in \mathbb{Z}}\left(\epsilon^{2 k}+\epsilon^{-2 k}\right)^{-s / 2} \sum_{\mu \in(\Lambda \backslash\{0\}) / V}|N(\mu)|^{-s / 2},
$$

using the unique decomposition $\lambda=A_{\epsilon}^{k}(\mu)$, for $k \in \mathbb{Z}$ and $\mu \in \mathcal{F}_{V}$, associated to the choice of the fundamental domain. Thus, we see that the finite summability condition holds.

Definition 8.15. The eta function of a Lorentzian $\mathbb{K}$-spectral triple is the function

$$
\eta_{\mathcal{D}}(s):=\sum_{n} \operatorname{sign}\left(\left\langle U e_{n}, \mathcal{D}^{2} U e_{n}\right\rangle\right)\left|\left\langle U e_{n},\left|\mathcal{D}^{2}\right| U e_{n}\right\rangle\right|^{-s / 2},
$$

where the sum is over an orthonormal basis for the complement of the zero modes of $\left|\mathcal{D}^{2}\right|$ in the Hilbert space $\mathcal{V}_{\mathbb{R}, i}$.

The following result relates the Shimizu $L$-function to the Lorentz $\mathbb{K}$ spectral triple. 
Corollary 8.16. The eta function for the Lorentz $\mathbb{K}$-spectral triple of Proposition 8.14 is of the form

$$
\eta_{\mathcal{D}_{\mathbb{K}}}(s)=L\left(\Lambda, V, \frac{s}{2}\right) Z_{\epsilon}\left(\frac{s}{2}\right),
$$

where $L(\Lambda, V, s)$ is the Shimizu L-function and $Z_{\epsilon}(s / 2)=\sum_{k \in \mathbb{Z}}\left(\epsilon^{2 k}+\epsilon^{-2 k}\right)^{-s / 2}$.

Proof. The argument is the same as in Proposition 8.14. We have

$$
\begin{aligned}
\sum_{\lambda \neq 0} & \operatorname{sign}\left(\left\langle U_{\epsilon} e_{\lambda, \pm}, \mathcal{D}_{\mathbb{K}}^{2} U_{\epsilon} e_{\lambda \pm}\right\rangle\right)\left|\left\langle U_{\epsilon} e_{\lambda, \pm},\left|\mathcal{D}_{\mathbb{K}}^{2}\right| U_{\epsilon} e_{\lambda, \pm}\right\rangle\right|^{-s / 2} \\
= & \sum_{\lambda \neq 0} \operatorname{sign}(N(\lambda))\left(\epsilon^{2 \rho(\lambda)}+\epsilon^{-2 \rho(\lambda)}\right)^{-s / 2}|N(\lambda)|^{-s / 2} \\
= & \sum_{k \in \mathbb{Z}}\left(\epsilon^{2 k}+\epsilon^{-2 k}\right)^{-s / 2} \sum_{\mu \in(\Lambda \backslash\{0\}) / V} \operatorname{sign}(N(\mu))|N(\mu)|^{-s / 2} .
\end{aligned}
$$

The result then follows since $L(\Lambda, V, s)=\sum_{\mu \in(\Lambda \backslash\{0\}) / V} \operatorname{sign}(N(\mu))|N(\mu)|^{-s}$.

\subsection{Eta function and 3-dimensional geometry}

The zeta and eta functions we obtained in Proposition 8.14 and Corollary 8.16 for the Lorentzian spectral geometry are closely related to those one can obtain from the spectral geometry of the 3-dimensional solvmanifold $X_{\epsilon}$ and the signature operator on the noncommutative torus.

We have seen in Section 7.4 above that the Dirac operator $\not_{X_{\epsilon}}$ on the 3-dimensional solvmanifold $X_{\epsilon}$ can be related to the signature operator $\not_{\theta, \theta^{\prime}}$ of (7.33) on the noncommutative torus $\mathbb{T}_{\Lambda, i}$. Up to a unitary equivalence, we have written in (7.36) the operator $\not_{\theta, \theta^{\prime}}$ in terms of the operators $\tilde{D}_{\theta, \theta^{\prime}}^{\mu}=$ $D_{\theta}^{\mu} B_{\theta}$, with $\mu \in(\Lambda \backslash\{0\}) / V$, defined as in (7.37).

The zeta and eta functions for the operator $\tilde{D}_{\theta, \theta^{\prime}}^{\mu}$ have the following form.

Lemma 8.17. The operator $\tilde{D}_{\theta, \theta^{\prime}}$ has an associated zeta function of the form

$$
\zeta_{\tilde{D}_{\theta, \theta^{\prime}}}(s)=2 Z_{\epsilon}\left(\frac{s}{2}\right) \sum_{\mu \in(\Lambda \backslash\{0\}) / V}|N(\mu)|^{-s / 2} .
$$


The eta function of $\tilde{D}_{\theta, \theta^{\prime}}$ vanishes due to the symmetry in the spectrum. However, the restriction $\tilde{D}_{\theta, \theta^{\prime}}^{+}$of $\tilde{D}_{\theta, \theta^{\prime}}$ to the subspace $\mathcal{H}^{+}$of the positive modes of the operator $B_{\theta}$ has a nonvanishing eta function of the form

$$
\eta_{\tilde{D}_{\theta, \theta^{\prime}}^{+}}(s)=L\left(\Lambda, V, \frac{s}{2}\right) Z_{\epsilon}\left(\frac{s}{2}\right)=\eta_{\mathcal{D}_{\mathbb{K}}}(s) .
$$

Proof. The operator

$$
B_{\theta}=\left(\begin{array}{cc}
0 & \epsilon^{-k}-i \epsilon^{k} \\
\epsilon^{-k}+i \epsilon^{k} & 0
\end{array}\right)
$$

has spectrum

$$
\operatorname{Spec}\left(B_{\theta}\right)=\left\{ \pm\left(\epsilon^{2 k}+\epsilon^{-2 k}\right)^{1 / 2}\right\}
$$

which is symmetric around zero. Thus, for the zeta function, we have

$$
\zeta_{\tilde{D}_{\theta, \theta^{\prime}}}(s)=\sum_{\mu \in(\Lambda \backslash\{0\}) / V}|N(\mu)|^{-s / 2} 2 \sum_{k \in \mathbb{Z}}\left(\epsilon^{2 k}+\epsilon^{-2 k}\right)^{-s / 2},
$$

while the eta function vanishes.

One can restrict the spectral triple for the noncommutative torus $\mathbb{T}_{\Lambda, i}$ to the subspace $\mathcal{H}^{+}$of the positive modes of the operator $B_{\theta}$, since the action of the $R_{\eta}^{\sigma}$ preserves this decomposition. The new Dirac operator $\tilde{D}_{\theta, \theta^{\prime}}^{+}$is then given by the restriction of $\tilde{D}_{\theta, \theta^{\prime}}$ to $\mathcal{H}^{+}$. It has a corresponding decomposition

$$
\tilde{D}_{\theta, \theta^{\prime}, 0}^{+}=\sum_{\mu \in(\Lambda \backslash\{0\}) / V} D_{\theta}^{\mu} B_{\theta}^{+},
$$

where $\operatorname{Spec}\left(B_{\theta}^{+}\right)=\left\{\left(\epsilon^{2 k}+\epsilon^{-2 k}\right)^{1 / 2}\right\}$. Thus, in this case, one obtains

$$
\eta_{\tilde{D}_{\theta, \theta^{\prime}}^{+}}(s)=L\left(\Lambda, V, \frac{s}{2}\right) \zeta_{B_{\theta}^{+}}(s)
$$

where

$$
\zeta_{B_{\theta}^{+}}(s)=Z_{\epsilon}\left(\frac{s}{2}\right)=\sum_{k}\left(\epsilon^{2 k}+\epsilon^{-2 k}\right)^{-s / 2}
$$

\subsection{The residue}

The special value $L(\Lambda, V, 0)$ of the Shimizu $L$-function can be extracted from the eta function $\eta_{\tilde{D}_{\theta, \theta^{\prime}}^{+}}(s)$ in the following way. 
Corollary 8.18. The function $\eta_{\tilde{D}_{\theta, \theta^{\prime}}^{+}}(s)$ has a pole of order 1 at $s=0$ with

$$
\operatorname{Res}_{s=0} \eta_{\tilde{D}_{\theta, \theta^{\prime}}^{+}}(s)=\frac{L(\Lambda, V, 0)}{\log \epsilon} .
$$

Proof. Consider the function

$$
Z_{\epsilon}(s):=\sum_{k \in \mathbb{Z}}\left(\epsilon^{2 k}+\epsilon^{-2 k}\right)^{-s} .
$$

It suffices to show that it has a simple pole at $s=0$ with residue

$$
\operatorname{Res}_{s=0} Z_{\epsilon}(s)=\frac{1}{\log \epsilon} .
$$

One writes

$$
\Gamma(s) Z_{\epsilon}(s)=\int_{0}^{\infty} g_{\epsilon}(t) t^{s-1} d t
$$

where

$$
g_{\epsilon}(t)=\left(\sum_{k \in \mathbb{Z}} e^{-\left(\epsilon^{2 k}+\epsilon^{-2 k}\right) t}\right)
$$

for $t>0$. The function $g_{\epsilon}(t)$ satisfies

$$
g_{\epsilon}(t)=-e^{-2 t}+2 h_{\epsilon}(t)-2 \sum_{k=0}^{\infty} e^{-\epsilon^{2 k} t}\left(1-e^{-\epsilon^{-2 k} t}\right) \text {, }
$$

where

$$
h_{\epsilon}(t)=\sum_{k=0}^{\infty} e^{-\epsilon^{2 k} t} .
$$

We can estimate $-e^{-2 t}=-1+O(t)$ when $t \rightarrow 0$ and $\left(1-e^{-\epsilon^{-2 k} t}\right)=$ $O\left(\epsilon^{-2 k} t\right)$, uniformly. Notice that

$$
h_{\epsilon}(t)-h_{\epsilon}\left(\epsilon^{2} t\right)=e^{-t}=\sum_{r=0}^{\infty} \frac{(-1)^{r}}{r !} t^{r}
$$

hence

$$
h_{\epsilon}(t)=\frac{1}{2 \log \epsilon} \log \left(\frac{1}{t}\right)+C-\sum_{r=0}^{\infty} \frac{(-1)^{r}}{r !\left(\epsilon^{2 r}-1\right)} t^{r} .
$$


Thus, the function $\Gamma(s) Z_{\epsilon}(s)$ has a double pole at $s=0$ and simple poles at $s \in \mathbb{Z}_{<0}$. Thus, the function $Z_{\epsilon}(s)$ has a simple pole at zero with residue $1 / \log \epsilon$.

\section{Acknowledgment}

I am very grateful to Sir Michael Atiyah for asking the question this paper is attempting to answer. I thank Alain Connes, Yuri Manin and Don Zagier for useful conversations. I also thank the Mittag Leffler Institute for hospitality and support, while part of this work was done. This research is partially supported by NSF grant DMS-0651925.

\section{References}

[1] M.F. Atiyah, Elliptic operators, discrete groups and von Neumann algebras, Colloque "Analyse et Topologie" en l'Honneur de Henri Cartan (Orsay, 1974), Asterisque, No. 32-33, Soc. Math., France, Paris, 1976, $43-72$.

[2] M.F. Atiyah, H. Donnelly and I.M. Singer, Eta invariants, signature defects of cusps, and values of L-functions, Ann. Math. 118 (1983), $131-177$.

[3] M.F. Atiyah, V.K. Patodi and I.M. Singer, Spectral asymmetry and Riemannian geometry. III, Math. Proc. Cambridge Philos. Soc. 79 (1) (1976), 71-99.

[4] P. Baum and A. Connes, Chern character for discrete groups, A fete of topology, Academic Press, Boston, MA, 1988, 163-232.

[5] J. Bellissard, Noncommutative geometry and quantum Hall effect, Proceedings of the International Congress of Mathematicians, Vol. 1, 2 (Zürich, 1994), Birkhäuser, 1995, 1238-1246.

[6] J. Bellissard, Gap labelling theorems for Schrödinger operators, From number theory to physics (Les Houches, 1989), Springer, Berlin, 1992, $538-630$.

[7] J. Bognár, Indefinite inner product spaces, Springer, 1974.

[8] J. Chabert and S. Echterhoff, Permanence properties of the BaumConnes conjecture, Doc. Math. 6 (2001), 127-183. 
[9] A. Connes, $C^{*}$-algèbres et géométrie différentielle, C. R. Acad. Sci. Paris Sér. A-B 290 (13) (1980), A599-A604.

[10] A. Connes, An analogue of the Thom isomorphism for crossed products of a $C^{*}$-algebra by an action of $\mathbb{R}$, Adv. Math. 39 (1) (1981), 31-55.

[11] A. Connes, Geometry from the spectral point of view, Lett. Math. Phys. 34 (3) (1995), 203-238.

[12] A. Connes, On the spectral characterization of manifolds, Preprint, 2008.

[13] A. Connes and G. Landi, Noncommutative manifolds, the instanton algebra and isospectral deformations, Comm. Math. Phys. 221 (1) (2001), 141-159.

[14] S. Echterhoff, W. Lück, N.C. Phillips and S. Walters, The structure of crossed products of irrational rotation algebras by finite subgroups of $S L_{2}(\mathbb{Z})$, Preprint SFB 478, Heft 435, 2006.

[15] E. Getzler, The odd Chern character in cyclic homology and spectral flow, Topology 32(3) (1993), 489-507.

[16] F. Hirzebruch, Hilbert modular surfaces, Enseignement Math. 19 (2) (1973), 183-281.

[17] D.G. Hofstadter, Energy levels and wave functions of Bloch electrons in rational or irrational magnetic field, Phys. Rev. B 14 (1976), 22392249 .

[18] Yu.I. Manin, Real multiplication and noncommutative geometry (ein Alterstraum), The legacy of Niels Henrik Abel, Springer, Berlin, 2004, 685-727.

[19] M. Marcolli and V. Mathai, Twisted index theory on good orbifolds, I: noncommutative Bloch theory, Commun. Contemp. Math. 1 (4) (1999), $553-587$.

[20] M. Marcolli and V. Mathai, Twisted index theory on good orbifolds, II: fractional quantum numbers, Commun. Math. Phys. 217 (2001), 55-87.

[21] M. Marcolli and V. Mathai, Towards the fractional quantum Hall effect, a noncommutative geometry perspective, Noncommutative Geometry and Number Theory, Vieweg Verlag, 2006, 235-262. 
[22] R. Matthes, O. Richter and G. Rudolph, Spectral triples and differential calculi related to the Kronecker foliation. J. Geom. Phys. 46 (1) (2003), 48-73.

[23] A. Mishchenko and A. Fomenko, The index of elliptic operators over $C^{*}$-algebras, Math. USSR Izv. 15 (1980), 87-112.

[24] W. Müller, Signature defects of cusps of Hilbert modular varieties and values of L-series at $s=1$, J. Diff. Geom. 20 (1) (1984), 55-119.

[25] J.A. Packer and I. Raeburn, On the structure of twisted group $C^{*}$ algebras, Trans. AMS 334 (1992), 685-718.

[26] A. Rennie and J. Varilly, Reconstruction of manifolds in noncommutative geometry, arXiv:math/0610418.

[27] M.A. Rieffel, $C^{*}$-algebras associated with irrational rotations, Pacific J. Math. 93 (2) (1981), 415-429.

[28] J. Rosenberg, $C^{*}$-algebras, positive scalar curvature, and the Novikov conjecture III, Topology 25 (1986), 319-336.

[29] A. Strohmaier, On noncommutative and pseudo-Riemannian geometry, J. Geom. Phys. 56 (2) (2006), 175-195.

[30] T. Sunada, A discrete analogue of periodic magnetic Schrödinger operators, Geometry of the spectrum (Seattle, WA, 1993), Contemp. Math., 173, Amer. Math. Soc., Providence, RI, 1994, 283-299.

MaX-Planck Institut Für Mathematik

Vivatsgasse 7, Bonn D-53111

GERMANY

E-mail address: marcolli@mpim-bonn.mpg.de

Received December 13, 2007 